\title{
Guidelines for the diagnosis, prevention and management of osteoporosis
}

\author{
M. Rossini, S. Adami, F. Bertoldo, D. Diacinti, D. Gatti, S. Giannini, \\ A. Giusti, N. Malavolta, S. Minisola, G. Osella, M. Pedrazzoni, \\ L. Sinigaglia, O.Viapiana, G.C. Isaia \\ On behalf of the Italian Society for Osteoporosis, Mineral Metabolism and Bone Diseases (SIOMMMS) \\ with the endorsement of \\ Italian Society of Endocrinology (SIE) \\ Italian Society of Geriatrics and Gerontology (SIGG) \\ Italian Society of Internal Medicine (SIMI) \\ Italian Society of Rheumatology (SIR)
}

\begin{abstract}
SUMMARY
Osteoporosis poses a significant public health issue. National Societies have developed Guidelines for the diagnosis and treatment of this disorder with an effort of adapting specific tools for risk assessment on the peculiar characteristics of a given population. The Italian Society for Osteoporosis, Mineral Metabolism and Bone Diseases (SIOMMMS) has recently revised the previously published Guidelines on the diagnosis, riskassessment, prevention and management of primary and secondary osteoporosis. The guidelines were first drafted by a working group and then approved by the board of SIOMMMS. Subsequently they received also the endorsement of other major Scientific Societies that deal with bone metabolic disease. These recommendations are based on systematic reviews of the best available evidence and explicit consideration of cost effectiveness. When minimal evidence is available, recommendations are based on leading experts' experience and opinion, and on good clinical practice. The osteoporosis prevention should be based on the elimination of specific risk factors. The use of drugs registered for the treatment of osteoporosis are recommended when the benefits overcome the risk, and this is the case only when the risk of fracture is rather high as measured with variables susceptible to pharmacological effect. DeFRA (FRAX ${ }^{\circledR}$ derived fracture risk assessment) is recognized as a useful tool for easily estimate the long-term fracture risk. Several secondary forms of osteoporosis require a specific diagnostic and therapeutic management.
\end{abstract}

Key words: Guidelines; risk factors; fractures; dual-energy x-ray absorptiometry; osteoporosis.

Reumatismo, 2016; 68 (1): 1-39

\section{INTRODUCTION} AND METHODOLOGY

\section{METHODS}

his document covers the guidelines for the diagnosis and management of both primary (postmenopausal and senile) and secondary osteoporosis. The recommendations were developed using a demonstrable and reproducible process for the evaluation of the bibliographic references, and result from a weighted evaluation of the level of evidence (Tables I-IV).

The recommendations were first prepared by a dedicated Committee and then reviewed and shared with a large number of representatives of general practitioners and representatives of various medical specialties involved in the diagnosis and prevention of osteoporosis, as well as with epidemiologists and health economics experts.

\section{Level of evidence}

\section{Criteria used to assign a level of evidence to articles}

\section{- Studies of treatment and intervention}

In recent years, registration studies of considerable size that included more subjects than previous meta-analyses have been conducted. For this reason, it was decided
Corresponding author Maurizio Rossini Rheumatology Unit P.co Borgo Roma P.le Scuro 10 - 37134 Verona E-mail: maurizio.rossini@univr.it 
Table I - Studies of diagnosis.

\begin{tabular}{|c|l|}
\hline $\begin{array}{c}\text { Level of } \\
\text { evidence }\end{array}$ & Criteria \\
\hline 1 & i. Independent interpretation of test results \\
\hline & ii. Independent interpretation of the diagnostic standard \\
\hline & $\begin{array}{l}\text { iii. Selection of individuals suspected (but not proven) } \\
\text { to have the disease }\end{array}$ \\
\hline & iv. Reproducible description of the test and the diagnostic standard \\
\hline 2 & v. At least 50 individuals with and 50 individuals without the disease \\
\hline 3 & Meets 4 of the Level 1 criteria \\
\hline 4 & Meets 3 of the Level 1 criteria \\
\hline
\end{tabular}

Table II - Studies of treatment and intervention.

\begin{tabular}{|c|l|}
\hline $\begin{array}{c}\text { Level of } \\
\text { evidence }\end{array}$ & Criteria \\
\hline 1 & Systematic overview of meta-analysis of randomised controlled trials \\
\hline 2 & Randomised controlled trial that does not meet Level 1 criteria \\
\hline 3 & Non-randomised controlled trial or cohort study \\
\hline
\end{tabular}

Table III - Studies of prognosis.

\begin{tabular}{|c|l|}
\hline $\begin{array}{c}\text { Level of } \\
\text { evidence }\end{array}$ & Criteria \\
\hline 1 & $\begin{array}{l}\text { i. Inception cohort of patients with the condition of interest, } \\
\text { but free of the outcome of interest }\end{array}$ \\
\hline & ii. Reproducible inclusion and exclusion criteria \\
\hline & iii. Follow-up of at least $80 \%$ of participants \\
\hline & iv. Statistical adjustment for confounders \\
\hline 2 & v. Reproducible description of the outcome measures \\
\hline 3 & Meets criterion i and 3 of the 4 of the Level 1 criteria \\
\hline 4 & Meets criterion i and 2 of the 4 of the Level 1 criteria \\
\hline
\end{tabular}

Table IV - Grades of recommendation for clinical practice guidelines.

\begin{tabular}{|c|l|}
\hline Grade & Criteria \\
\hline A & $\begin{array}{l}\text { Need supportive level } 1 \text { or } 1+ \\
\text { evidence plus consensus }\end{array}$ \\
\hline B & $\begin{array}{l}\text { Need supportive level } 2 \text { or } 2+ \\
\text { evidence plus consensus }\end{array}$ \\
\hline C & $\begin{array}{l}\text { Need supportive level } 3 \\
\text { evidence plus consensus }\end{array}$ \\
\hline D & $\begin{array}{l}\text { Any lower level of evidence } \\
\text { supported by consensus }\end{array}$ \\
\hline
\end{tabular}

*An appropriate level of evidence was necessary, but not sufficient to assign a grade in recommendation; consensus was required in addition. not to assign a higher level of evidence to meta-analyses of independent trials.

The levels of evidence described above will be integrated with individual notes taking into account post-hoc analyses that, despite having limitations, were considered to be particularly convincing.

\section{DEFINITION}

Osteoporosis is a systemic skeletal disorder characterized by decreased bone mass and qualitative alterations (macro- and micro-architecture, bone material properties) associated with increased fracture risk. Primary osteoporosis is defined as osteoporosis occurring after menopause (postmenopausal osteoporosis) or with advancing age (senile osteoporosis). Secondary osteoporosis is caused by a number of disorders and drugs.

Bone densitometry allows quite accurate and precise measurement of bone mass, particularly its mineral density [bone mineral density (BMD)] in $\mathrm{g} / \mathrm{cm}^{2}$ of projected bone area. BMD accounts for 60 to $80 \%$ of bone mechanical resistance.

According to the World Health Organization (WHO), a densitometric diagnosis of osteoporosis should be based on BMD measured by dual-energy x-ray absorptiometry (DXA), compared to the mean BMD in young normal adults of the same sex (peak bone mass). The unit of measurement is the standard deviation (SD) above or below the mean peak bone mass (T-score). It has been reported that fracture risk begins to increase exponentially at a $\mathrm{T}$ score $<-2.5 \mathrm{SD}$, which has been established by the WHO as the cut-off for diagnosing osteoporosis. Bone densitometry is therefore the diagnostic test for osteoporosis and fracture risk assessment, just as blood pressure measurement is used to diagnose arterial hypertension and assess the risk of stroke.

According to the WHO, the following definitions should be used to interpret BMD measurement:

1) Normal BMD is defined as a T-score between +2.5 and -1 (the patient's BMD is between 2.5 SD above and 1 
SD below the mean for a young normal adult of the same sex).

2) Osteopenia (low BMD) is defined as a $\mathrm{T}$-score between -1.0 and $-2.5 \mathrm{SD}$.

3) Osteoporosis is defined as a T-score below -2.5 SD.

4) Severe osteoporosis is defined as a Tscore below $-2.5 \mathrm{SD}$ in the presence of one or more fragility fractures.

It should be noticed that this is just a densitometric diagnosis that can be translated into a clinical diagnosis only after an overall clinical evaluation and differential diagnosis.

Furthermore, diagnostic T-score threshold does not equate the intervention threshold, as other factors - both skeletal and non-skeletal - affect an individual's fracture risk and the decision of initiating drug treatment.

\section{EPIDEMIOLOGY}

Osteoporosis places a relevant burden on society. The incidence of osteoporosis increases with ageing, affecting most of the population after the eighth decade of life. It has been estimated that approximately 3.5 million women and 1 million men have osteoporosis in Italy. As the proportion of individuals over the age of 65 years will increase by $25 \%$ in the next 20 years in Italy, a proportional increase in osteoporosis incidence is expected (1).

The lifetime risk of osteoporotic fracture of the distal wrist, vertebral bodies or proximal femur is approximately $15 \%$ for each individual site and $40 \%$ for all sites.

The number of hip fractures in the Italian population aged 50 years or older is greater than 90,000 per year. Vertebral deformities have been found in over $20 \%$ of subjects aged 65 or older of both genders.

Osteoporotic fractures have important social and economic implications, besides the health burden. The 1-year mortality rate for patients with a fracture of the proximal femur is 15 to $30 \%$. Osteoporotic fractures are one of the leading causes of death among the elderly, with an incidence comparable to that from stroke and breast cancer, and 4-fold that from endometrial can- cer. Furthermore, in $50 \%$ of women with a hip fracture there is a substantial reduction in the level of independence, which leads to the need for long-term care in $20 \%$ of cases.

\section{RISK FACTORS}

The clinical relevance of osteoporosis is related to a reduction in bone strength that leads to an increase in the risk of fragility fractures from low-energy traumas. Bone resistance to trauma depends on both quantitative factors, such as BMD assessed by DXA, and qualitative factors, such as geometry, microstructure, inorganic and organic composition of the matrix, that are not routinely assessed in clinical practice. Low-energy fractures generally result from accidental falls from standing or simple activities of daily life. With falls, fracture probability depends on the characteristics of the fall, the effectiveness of protective responses, and the soft tissue attenuation of the impact energy. Clearly, factors that affect the risk of fall tend to increase the risk of fracture. Thus, multiple factors that affect both bone strength and the frequency and type of traumas are involved in the pathogenesis of fractures. The risk of osteoporotic fracture results from a combination of factors that act predominantly via a reduction in BMD and factors that are totally or partially independent from BMD (bone properties that cannot be assessed by BMD and extra skeletal factors). Clearly, this distinction is not clear-cut, and several risk factors act through different mechanism simultaneously. Among factors that are independently associated with both osteoporosis and fracture risk, age, prior fragility fractures, family history of fragility fractures, corticosteroid use and conditions that increase the likelihood of falls are of particular importance. Furthermore, the presence of comorbidities increases fracture risk. In subjects with several risk factors, fracture risk is higher than in subjects with a single risk factor, including an isolated reduction in BMD. Therefore, BMD measurement is adequate for diag- 
nosing osteoporosis (diagnostic threshold), but combining BMD with independent risk factors is necessary to identify individuals at high risk of fracture requiring a specific pharmacological or non-pharmacological intervention, whatever appropriate, according to the kind of risk factors mainly involved.

\section{Main risk factors}

- Bone mineral density

A reduction in BMD is an important risk factor for fractures. BMD is dependent on the peak bone mass achieved at maturity and the subsequent bone loss associated with menopause and ageing, and is affected by genetic and nutritional factors, lifestyle, comorbid conditions and a number of drugs (Table V). Several prospective epidemiological studies have shown that for every 1 SD decrease in BMD [mostly assessed by DXA of axial sites (i.e., femoral neck, total femur and lumbar spine)] there is a 1.5- to 3-fold increase in the risk of fracture. The ability of BMD to predict fractures is similar to that of blood pressure as a predictor of stroke, and better than that

Table V - Risk factors for osteoporosis and/or osteoporotic fractures.

\begin{tabular}{|l|}
\hline - Age \\
\hline - Female gender \\
\hline - Low body mass index \\
\hline $\begin{array}{l}\text { - Prior fragility fracture (particularly spine, including } \\
\text { morphometric fractures, wrist, hip and humerus) }\end{array}$ \\
\hline - Family history of hip/vertebral fracture \\
\hline - Cigarette smoking (current) \\
\hline - Alcohol intake (3 or more units per day) \\
\hline - Vitamin D deficiency \\
\hline - Early menopause (before the age of 45 years) \\
\hline - Low physical activity \\
\hline - Prolonged immobility \\
\hline - Reduced calcium intake \\
\hline - Excessive sodium intake \\
\hline - Osteoporosis-associated diseased \\
\hline - Organ transplant \\
\hline - Drugs \\
\hline
\end{tabular}

of cholesterol as a predictor of myocardial infarction. Nevertheless, despite BMD being an important risk factor for fracture, its predictive power increases when other independent risk factors that can provide additional and complementary information to BMD are considered.

- Age

With ageing, there is an exponential increase in the incidence of osteoporotic fractures. The risk of fracture associated with ageing is only in part mediated by a reduction in BMD, being largely dependent on other factors, such as qualitative alterations of bone structure, an increase in the rate of falls and slower protective responses. The same T-score has a different significance at different ages, and for a given BMD the risk of fracture is higher in the elderly than in the young.

\section{- Previous fractures}

A previous fragility fracture is a strong risk factor for future fractures in both genders. Even though a previous fracture is often associated with reduced BMD, the risk for new fractures is largely independent of BMD.

Recent epidemiological studies showed that having a previous fracture of any site increases the risk of new fractures, although to a different extent depending on the site. Vertebral fractures (including morphometric fractures), as well as fractures of the wrist and humerus are of particular prognostic relevance (2-4). Furthermore, the risk is dependent on the number of previous fractures. The risk of new fractures in individuals with 3 or more fractures is approximately 10 -fold higher compared to that in individuals with no fractures, and 2to 3-fold higher compared to those with a single fracture $(2,5)$.

\section{- Family history}

Fracture risk is affected by family history of fracture independently of BMD. Specifically, having a parent with a hip fracture significantly increases the risk of hip fracture and, although to a lesser extent, of all osteoporotic fractures $(6,7)$.

\section{- Comorbidities}

Several disorders are associated with an increased fracture risk (Table V). The 
Table VI - Risk factors for falls.

\begin{tabular}{|l|}
\hline - Individual factors \\
\hline - History of previous falls \\
\hline - Conditions that impair \\
\hline Muscle mass/strength (sarcopenia) \\
\hline Lower limb function \\
\hline Balance \\
\hline Vision \\
\hline - Cognitive impairment \\
\hline - Arrhythmias \\
\hline - Drugs (drugs that act on the CNS, \\
anti-hypertensives, alcohol) \\
\hline - Muscle impairment due to hypovitaminosis D \\
\hline - Environmental factors \\
\hline - Barriers, lighting, flooring, shoes \\
\hline
\end{tabular}

CNS, Central nervous system.

risk is thought to be mediated by a reduction in BMD in many of these conditions. However, different mechanisms, such as chronic inflammation, altered bone quality, impaired health status, specific complications, decreased mobility, decreased muscle mass/strength (sarcopenia) and increased risk of falls may also play a role (Table VI). Vitamin D deficiency often coexists, representing a further negative factor. Comorbidities to be mentioned include rheumatoid arthritis and connective tissue diseases, diabetes, chronic obstructive pulmonary disease, chronic inflammatory bowel diseases, AIDS, Parkinson's disease, multiple sclerosis, conditions associated with severe motor disability. Of note, individuals with type 1 or type 2 diabetes tend to fracture at a higher BMD as compared to non-diabetic subjects, therefore the risk of fracture is partially independent of BMD in this population (7-15).

\section{- Drugs}

Several drugs have been associated with an increased fracture risk. The detrimental effects of corticosteroids on bone are well known. Although these are mainly mediated by factors independent of bone density, corticosteroid treatment also leads to a rapid reduction in BMD. Among recent drugs, adjuvant hormone antagonist thera- pies [aromatase inhibitors in women who have undergone surgery for breast cancer, gonadotropin releasing hormone $(\mathrm{GnRH})$ agonists in men with prostate cancer] need special attention. Treatment with these drugs results in a progressive decrease in BMD, although a role for independent factors for fracture risk cannot be excluded.

\section{Overall assessment of fracture risk}

An integrated assessment of BMD in conjunction with the main clinical risk factors that are totally or partially independent of BMD allows for a more accurate estimate of the risk for fragility fractures in the medium term (5 to 10 years), and to identify subjects requiring pharmacological or non-pharmacological treatment, whatever appropriate. In the past decade several algorithms, e.g. FRAX® and DeFRA®, have been developed that estimate the 10-year risk of a major osteoporotic fracture (spine, hip, humerus or wrist fracture) by integrating information from BMD measurement and clinical risk factors. The identification of clinical risk factors independent of BMD to be included in these algorithms was based on a number of studies and meta-analyses, as well as on their ease of identification and quantification. Risk assessment, whether using or not these algorithms, should be based on easily available information. When an algorithm includes too many variables, such as the QFracture, or variables that are not easily available, chances are that the algorithm or tool is not implementable in clinical practice due to its complexity, which would make it completely useless. On the other hand, however, when the definition of risk is based on few factors, maybe not even stratified - as in the Garvan fracture risk calculator - it is possible that the algorithm and risk stratification lack in accuracy, thereby not providing specific advantages over BMD measurement.

Based on this background, and in order to overcome some of the limitations of FRAX ${ }^{\circledR}$ as well as to improve fracture risk prediction, the Italian DeFRA® - an algorithm derived from FRAX ${ }^{\circledR}$ and based on data on fracture risk in the Italian popula- 
tion - was developed that more accurately stratifies some of the variables already included in FRAX® (e.g. site and number of previous fractures, comorbidities). Given their great relevance, clinical risk factors independent of BMD have also been used in the definition of criteria for the reimbursement of drugs for the treatment of osteoporosis in Italy [Italian Medicines Agency (AIFA) Note 79]. The Note 79 was recently revised, which highlights the fact that tools for the assessment of fracture risk should be constantly and frequently updated with data from clinical studies that may identify new clinical risk factors (as in the case of diabetes and of aromatase inhibitors), or may allow for a better interpretation of risk based on known factors.

\section{GENETICS OF OSTEOPOROSIS}

Genes appear to be the main determinant of person-to-person variability in bone mass. Propensity to osteoporosis, similar to many other disorders, is attributable to the overall combined effect of several gene polymorphisms.

Polymorphisms of genes encoding type 1 collagen (COLIA1), estrogen receptor (ER) and vitamin D receptor (VDR) are currently regarded as possible genetic determinants of osteoporosis risk.

Each of these polymorphisms can explain less than $30 \%$ of variability in bone mass, and even less of the risk of developing osteoporosis. Diagnostic and prognostic use of gene polymorphisms is therefore not justified.

\section{SECONDARY OSTEOPOROSIS}

Postmenopausal/senile osteoporosis should always be distinguished from secondary osteoporosis. A number of factors have the potential to induce osteoporosis, including several diseases and drugs (Table VII). Recommendations for the management of the most typical or frequent forms of secondary osteoporosis are provided below, focusing on the specific diagnostic and therapeutic aspects related to osteoporosis.

\section{PRYMARY HYPERPARATHYROIDISM}

\section{Definition. Epidemiology}

Primary hyperparathyroidism (PHPT) is a disorder of calcium and phosphorus metabolism caused by an autonomous and relatively uncontrolled secretion of parathyroid hormone (PTH) by one or more hyperfunctioning parathyroid glands, resulting in hypercalcaemia. PHPT, together with hypercalcaemia of malignancy, accounts for $90 \%$ of all hypercalcaemic states. PHPT is a very common endocrine disease, with an incidence of approximately 25/100,000 persons-year in the male US population and $65 / 100,000$ persons-year in the female US population; age-corrected prevalence is $85 / 100,000$ in men and $233 / 100,000$ in women (16). PHPT is more common in women (the female/male ratio is approximately $2: 1$, and increases up to $5: 1$ after the age of 75 years) (17). In most cases PHPT is caused by a single parathyroid adenoma (75-85\%), whereas $15-20 \%$ of cases are caused by hyperplasia affecting more than one parathyroid gland. The finding of multiple adenomas is less frequent, and malignancies are rare (less than $1 \%$ of cases) (17). Approximately $10 \%$ of cases are familial, occurring as part of a multiple endocrine neoplasia (MEN1, MEN2A, other rare forms). Sometimes an adenoma may arise from an ectopic parathyroid gland (mainly in the mediastinum).

\section{Diagnosis and differential diagnoses}

PHPT should be suspected in all cases of hypercalcaemia, being the most common cause of such metabolic alteration in outpatients. The diagnosis is primarily biochemical, and is based on the finding of hypercalcaemia and PTH levels that are high or inappropriately normal relative to serum calcium levels. Near-normal calcium levels may be found in mild PHPT (normocalcaemic primary hyperparathyroidism). In such cases, calcium levels should be measured several times and albumin-corrected calcium levels should be calculated (18). Furthermore, pharmacological causes (e.g., thiazide diuretics, lithium salts bisphos- 
Table VII - Diseases associated with osteoporosis.

\begin{tabular}{|c|c|}
\hline Endocrine disorders & Kidney diseases \\
\hline \multirow{3}{*}{$\begin{array}{l}\text { Hypogonadism } \\
\text { Hypercortisolism } \\
\text { Hyperparathyroidism } \\
\text { Hyperthyroidism } \\
\text { Hyperprolactinaemia } \\
\text { Diabetes mellitus type } 1 \text { and } 2 \\
\text { Acromegaly } \\
\text { GH deficiency }\end{array}$} & $\begin{array}{l}\text { Idiopathic renal hypercalciuria } \\
\text { Renal tubular acidosis } \\
\text { Chronic kidney disease }\end{array}$ \\
\hline & Neurologic disorders \\
\hline & \multirow{3}{*}{$\begin{array}{l}\text { Parkinson's disease } \\
\text { Multiple sclerosis } \\
\text { Paraplegia } \\
\text { Sequelae of stroke } \\
\text { Muscular dystrophies }\end{array}$} \\
\hline Haematologic disorders & \\
\hline \multirow{3}{*}{$\begin{array}{l}\text { Myelo- and lymphoproliferative diseases } \\
\text { Multiple myeloma } \\
\text { Systemic mastocytosis } \\
\text { Thalassemia } \\
\text { Monoclonal gammopathies } \\
\text { Sickle cell anaemia } \\
\text { Haemophilia }\end{array}$} & \\
\hline & Genetic disorders \\
\hline & \multirow{3}{*}{$\begin{array}{l}\text { Osteogenesis Imperfecta } \\
\text { Ehlers-Danlos } \\
\text { Gaucher's disease } \\
\text { Glycogenosis } \\
\text { Hypophosphatasia } \\
\text { Haemochromatosis } \\
\text { Homocystinuria } \\
\text { Cystic fibrosis } \\
\text { Marfan syndrome } \\
\text { Menkes syndrome } \\
\text { Porphyria } \\
\text { Riley-Day syndrome }\end{array}$} \\
\hline Gastrointestinal disorders & \\
\hline \multirow[t]{2}{*}{$\begin{array}{l}\text { Chronic liver disease } \\
\text { Primary biliary cirrhosis } \\
\text { Celiac disease } \\
\text { Chronic inflammatory bowe } \\
\text { Gastrointestinal resection } \\
\text { Gastric bypass } \\
\text { Lactose intolerance } \\
\text { Intestinal malabsorption } \\
\text { Pancreatic insufficiency }\end{array}$} & \\
\hline & Other disorders \\
\hline Rheumatic disorders & Chronic obstructive pulmonary disease \\
\hline $\begin{array}{l}\text { Rheumatoid arthritis } \\
\text { Systemic lupus erythematosus } \\
\text { Ankylosing spondylitis } \\
\text { Psoriatic arthritis } \\
\text { Scleroderma } \\
\text { Other connective tissue diseases }\end{array}$ & $\begin{array}{l}\text { Anorexia nervosa } \\
\text { AIDS/HIV } \\
\text { Amyloidosis } \\
\text { Sarcoidosis } \\
\text { Depression }\end{array}$ \\
\hline
\end{tabular}

phonates) should be ruled out, and hypovitaminosis D, when present, should be corrected. Measurement of ionized calcium is justified only when performed in optimal technical conditions. Assays that measure intact PTH should be preferred. The finding of reduced 24-h urine calcium mandates exclusion of familial hypocalciuric hypercalcemia (FHH), i.e., the other hypercalcaemic state (along with use of lithium salts or thiazide diuretics) where elevated PTH levels are seen.

As parathyroidectomy is not required and does not correct hypercalcaemia in $\mathrm{FHH}$ patients, it is important to make the correct differential diagnosis. A ratio between fractional excretion of calcium and creatinine clearance of $<0.01$ is typical of $\mathrm{FHH}$ patients, and is useful to differentiate them from patients with PHPT (19).

Preoperative localization of the adenoma or hyperplastic parathyroid [by ultrasonography, sestamibi scintigraphy, or multidimensional computed tomography (CT)] is not indicated during the diagnostic process. The usefulness of preoperative localization once the indication for surgery has been established (although preoperative localization is often used to facilitate surgical exploration) is debated, whereas localization is necessary prior to reoperation in patients with persistent PHPT (18).

\section{Clinical manifestations}

In most instances, PHPT is diagnosed in the absence of symptoms. Severe mani- 
festations typical of PHPT (osteitis fibrosa cystica, nephrolithiasis- nephrocalcinosis, bone brown tumours) have become very rare since measurement of serum calcium has become routine clinical practice, as of the 1970s, allowing for an early diagnosis of disease. Kidney stones are present in approximately $10 \%$ of subjects at diagnosis, and are related to hypercalciuria; the incidence of US-detected stones is higher. Besides the risk of kidney stones, patients with overt PHPT are at increased risk of fracture, due to a reduction in bone mass (particularly in cortical bone, but also in trabecular bone). Surgical treatment of PHPT can reduce the recurrence of kidney stones, as well as the risk of fracture $(20,21)$. Given its high frequency, PHPT should always be considered as a possible cause of osteoporosis. As such, measurement of serum calcium should always be included among the first tier investigations to be performed in a patient with reduced bone mass. An acute hypercalcaemic crisis (parathyroid crisis) is a very rare presentation of PHPT. It is facilitated by dehydration and characterized by symptoms of severe hypercalcaemia, and is potentially deadly. Although an exceptional onset, an hypercalcaemic crisis should be considered in any patient with severe hypercalcaemia of unclear etiology.

\section{Treatment}

\section{- Surgical treatment}

In the absence of contraindications, surgery is the definitive and causal treatment of symptomatic PHPT (nephrolithiasis, symptomatic hypercalcaemia). The surgical procedure consists of neck exploration to identify all 4 parathyroid glands, and removal of the one(s) with adenomatous or hyperplastic features. The surgeon's experience and intraoperative PTH measurement are important in reducing the risk of persistent PHPT. Minimally invasive surgery of parathyroid glands (unilateral surgery based on scintigraphy) in conjunction with intraoperative PTH measurement is safe and can be as effective as bilateral neck exploration under general anaesthesia (Level of evidence 2). The role of surgery in the management of asymptomatic PHPT in patients aged $>50$ years is not clearly established. With regard to the indications for parathyroidectomy, the present guidelines are in agreement with recommendations from the Fourth International Workshop on this topic, issued in 2014 (18):

- Serum calcium concentration $\geq 1.0 \mathrm{mg} /$ $\mathrm{dL}(0.25 \mathrm{mmol} / \mathrm{L})$ above the upper limit of normal.

- Estimated glomerular filtration rate $<60$ $\mathrm{mL} / \mathrm{min}$

- BMD T-score $<-2.5$ at the distal radius or spine and/or previous asymptomatic vertebral fractures (assessed by x-ray, CT, MRI or DXA morphometry)

- 24-hour urinary calcium $>400 \mathrm{mg}$ per day (>10 mmol/day)

- Nephrolithiasis or nephrocalcinosis diagnosed by $\mathrm{x}$-ray, ultrasound or CT Age $<50$ years

Surgery is also indicated for patients who cannot be followed up or in case of patient preference for surgery, even if the above indications are not met, and in the absence of contraindications.

In patients who do not meet the criteria for parathyroidectomy, supportive and preventive measures in conjunction with adequate monitoring should be implemented (18):

- Measurement of serum calcium, creatinine and estimated glomerular filtration rate yearly.

- DXA (of all 3 sites) every 1 to 2 years.

- 24-hour urinary calcium, abdominal $\mathrm{x}$-ray or ultrasound only when kidney stones are suspected.

If during follow up a change in the clinical picture leads to satisfaction of the above criteria, the patient should be addressed to surgery.

General recommendations for patients not undergoing surgery include: i) avoid factors that may aggravate hypercalcaemia [thiazide diuretics, lithium salts, volume depletion, bed confinement or inactivity, and excessive calcium dietary intake (>1000 mg per day)]; ii) maintain an adequate intake of calcium (800-1000 mg per day) and vitamin D (400-800 IU/day, in order to maintain serum hydroxy-vitamin 
D levels of at least 20-30 ng/mL), avoiding hydroxylated derivatives; iii) physical activity should be encouraged; iv) adequate hydration (at least 6-8 glasses of water per day) should be encouraged to reduce the risk of nephrolithiasis.

\section{- Medical therapy}

Medical therapy for the control of severe hypercalcaemia is indicated in patients with contraindications to surgery or in preparation for surgery, and consists of adequate hydration with saline (2-4 L/day for 1-3 days on average), use of loop diuretics (furosemide) once volume has been restored, and use of i.v. bisphosphonates (e.g., a single dose of zoledronic acid $4 \mathrm{mg}$ or pamidronate $60-90 \mathrm{mg}$ ). If quick correction of calcium levels is required (e.g. in the case of parathyroid crisis), administration of 4 $\mathrm{U} / \mathrm{Kg}$ of i.m. or s.c. calcitonin can be considered. If surgery for parathyroidectomy is scheduled within few days, i.v. bisphosphonates should be avoided (for possible worsening of postoperative hypocalcaemia). Although direct evidence is lacking (level 3), it is reasonable to recommend use of oral bisphosphonates (alendronate) if osteoporosis is present. Hormone replacement therapy or raloxifene may be indicated in postmenopausal women who have no contraindications. Further studies are necessary to establish the long-term benefit of cinacalcet, a calcimimetic that selectively inhibits PTH secretion. This drug is approved both for the treatment of secondary hyperparathyroidism in patients with endstage renal disease on maintenance dialysis therapy and for the reduction of hypercalcaemia in patients with parathyroid carcinoma or patients with PHPT for whom parathyroidectomy would be indicated, but in whom parathyroidectomy is not clinically appropriate or is contraindicated.

\section{GLUCOCORTICOID-INDUCED OSTEOPOROSIS}

Epidemiology and clinical manifestations Chronic exposure to glucocorticoids, either due to increased endogenous production (Cushing's syndrome) or exogenous intake (treatment of inflammatory or autoimmune diseases) is an important cause of osteoporosis and fractures. In fact, glucocorticoids stimulate bone resorption by osteoclasts, reduce bone formation by inhibiting osteoblast proliferation and differentiation as well as by favouring osteoblast and osteocyte apoptosis, alter calcium metabolism by reducing intestinal calcium absorption and by increasing renal excretion, and reduce androgen and estrogen secretion mainly by inhibiting pituitary gonadotropin secretion (22). Glucocorticoid-induced bone loss is an early event (in the first weeks of treatment), and is more pronounced during the first 6 to 12 months of treatment, mainly affecting trabecular bone (particularly, vertebral fractures occur early after initiation of steroid therapy). Thereafter, there is a reduction in osteoclast-mediated bone resorption but inhibition of bone formation is maintained: bone loss is slower but constant, also involving cortical bone $(22,23)$. Fragility fractures occur in 30 to $50 \%$ of patients in the first 5 years of long-term glucocorticoid therapy. Fracture probability is further increased in the presence of other risk factors, particularly advanced age, previous fractures and menopause in women. Fractures may occur at any skeletal site, with predominance at sites with predominantly trabecular bone: spine, rib, and proximal femur (22). The incidence of fractures is related to the dose and duration of glucocorticoid therapy, and is also affected by the underlying disease for which glucocorticoid therapy is indicated (e.g., rheumatoid arthritis, inflammatory bowel disease). Although low doses are less detrimental than higher doses, there is controversy as to whether there is a threshold below which no bone damage occurs. There is also much controversy about the negative impact of inhaled glucocorticoids on bone health. Inhaled glucocorticoids are certainly less detrimental to bone as compared to systemic glucocorticoids, although doses $>800 \mathrm{mcg} /$ day of budesonide (or equivalents) may be associated with accelerated bone loss and increased risk of fractures, especially with long-term use (24-26). It is important to remember that fracture risk 
in glucocorticoid-induced osteoporosis is much higher as compared to that expected based on DXA BMD values, and rapidly decreases after treatment discontinuation $(27,28)$. Both of these observations have a practical implication: prevention should be started as early as possible, independent of BMD values, and prior to the occurrence of irreversible alterations of bone microarchitecture.

\section{Treatment}

- Identification of patients to be tested and treated

Most guidelines set the threshold for intervention at a dose of $7.5 \mathrm{mg}$ per day of prednisone (or equivalent doses of other glucocorticoids). However, it is known that doses between 2.5 and $7.5 \mathrm{mg}$ per day of prednisone are associated with an increased risk of fracture. Besides the daily dose, duration of glucocorticoid therapy appears to be very important. Fracture risk increases significantly after only 3 months of treatment, and decreases rapidly after discontinuation of treatment. Glucocorticoid-induced bone damage is largely independent of bone mass, and the lumbar spine T-score threshold to identify patients who need prevention and treatment is set between -1.5 and -1.0 (which equals to state that risk is essentially independent of bone mass, and is significant even for a T-score in the normal range).

The present guidelines recommend pharmacological treatment for the prevention of glucocorticoid-induced osteoporosis in postmenopausal women or men aged $\geq 50$ years who are receiving or planning to receive glucocorticoid therapy with $\geq 5 \mathrm{mg}$ prednisone or equivalent for $\geq 3$ months.

- Interventions with proven efficacy

1. General measures: glucocorticoid therapy should be reduced to the lowest possible dose and duration compatible with the need of controlling the underlying disease. Whenever possible, topical formulations (e.g., inhaled steroids or enemas for asthma and inflammatory bowel diseases, respectively) should be preferred over oral or parenteral administration. Patients should be encouraged to take adequate weightbearing exercise to counteract the loss of bone and muscle mass. Cigarette smoking and excessive alcohol consumption should be avoided, and measures to reduce the risk of falls should be considered (29) (Grade A recommendation).

2. Calcium and vitamin D: supplementation with 1000-2000 mg per day of calcium and 500-800 International Units (IU) per day of vitamin D was shown effective in decreasing glucocorticoid-induced bone loss (30) (Level 1b). Supplementation with hydroxylated metabolites of vitamin D does not appear to offer additional advantages over cholecalciferol. As in the studies that showed the anti-fracture efficacy of bisphosphonates patients were also supplemented with calcium and vitamin D, these should be recommended for all patients treated or planned for treatment with bisphosphonates (Grade A recommendation).

3. Hormone replacement therapy: glucocorticoids reduce the production of sex hormones. As such, it appears rationale to replace sex hormones in women with amenorrhea and estrogen deficiency (hypothalamic amenorrhea or primary ovarian insufficiency) or in men with proven hypogonadism $(31,32)$. While estrogen therapy is no longer considered a first line therapy for the prevention of postmenopausal osteoporosis, it appears reasonable - in the absence of contraindications - to consider hormone replacement therapy in premenopausal women with estrogen deficiency or in men with hypogonadism treated with glucocorticoids, even if controlled studies on the incidence of fractures are lacking (Grade A recommendation).

4. Bisphosphonates: bisphosphonates, particularly alendronate, risedronate and zoledronate, are the most widely used drugs for the prevention and treatment of glucocorticoid-induced osteoporosis in postmenopausal women and men. Randomized, controlled trials are available on these bisphosphonates (Level of evidence 1a), although underpowered to detect a reduction in fractures as a primary endpoint 
(33-35). The efficacy of bisphosphonates in reducing fractures can be inferred from meta-analyses or post-hoc analyses $(36,37)$ (Level of evidence 2). Therefore, bisphosphonate therapy in postmenopausal women and in men should be recommended as first line for the primary prevention, and should be started at the beginning of glucocorticoid therapy (Grade A recommendation).

5. Teriparatide: in a 36-month study that compared teriparatide versus alendronate in patients treated with glucocorticoids, teriparatide was more effective than alendronate in improving BMD (primary endpoint) and incidence of vertebral fractures (secondary endpoint) (38). For this reason, treatment with teriparatide is considered as a first line option for patients with glucocorticoid-induced osteoporosis and at least one previous fracture (secondary prevention) in the recent revision of the Italian AIFA Note 79. Treatment with teriparatide, as per the summary of characteristics, should not exceed 24 months. After this time, if glucocorticoid therapy is continued and fracture risk persists, patients should be switched to antiresorptive therapy to maintain the anti-fracture effect and the gain in terms of BMD.

6. Denosumab: in a post-hoc analysis of a randomized controlled study in patients with rheumatoid arthritis (some of them treated with glucocorticoids), denosumab was shown to induce a significant increase in lumbar spine and hip BMD versus placebo (39). In the AIFA Note 79 denosumab is considered as a second-line therapy both for primary and secondary prevention in patients treated with glucocorticoids.

\section{OSTEOPOROSIS RELATED TO OTHER DRUGS}

Several drug classes have been associated with osteoporosis and fragility fractures. Many of these data come from epidemiological and retrospective studies, and the level of evidence is often very low. Furthermore, a clear pathophysiological rationale is lacking for many of these associations, and is not known for some (Table VIII) (40-42). Besides glucocorticoid therapy and aromatase and GnRH inhibitors, which are treated separately in these guidelines,

Table VIII - Drugs associated with bone loss.

\begin{tabular}{|c|c|c|}
\hline Drug class & Active substance & Possible mechanism of action \\
\hline Glucocorticoids* & Hydrocortisone, prednisone, dexamethasone & Inhibition of osteoblast activity/osteocyte apoptosis \\
\hline Aromatase inhibitors* & Letrozole, anastrozole, examestane & Hypogonadism with high turnover \\
\hline SSRIss* & Citalopram, fluoxetine, paroxetine & Inhibition of osteoblast proliferation, RANKL activation \\
\hline Proton pump inhibitors* & Esomeprazole, omeprazole, lansoprazole & Reduced calcium intestinal absorption \\
\hline H2-inhibitors & Ranitidine, cimetidine & Reduced calcium absorption \\
\hline Thiazolidinediones* & Rosiglitazone, pioglitazone & $\begin{array}{l}\text { Inhibition of bone formation and osteoblast } \\
\text { differentiation }\end{array}$ \\
\hline Thyroid hormone (excess)* & Levothyroxine & Increased bone turnover \\
\hline Anticoagulants* & Heparin, warfarin & Reduced osteocalcin activity \\
\hline Anticonvulsants* & Phenobarbital, valproic acid, oxcarbazepine, phenytoin & Altered vitamin D metabolism \\
\hline $\mathrm{GnRH}^{*}$ & Leuprolide, goserelin & Hypogonadism with high turnover \\
\hline Loop diuretics & Furosemide & Calciuric effect \\
\hline Antiretroviral agents & $\begin{array}{l}\text { Efavirenz, nevirapine } \\
\text { Tenofovir } \\
\text { Protease inhibitors }\end{array}$ & $\begin{array}{l}\text { Altered vitamin D metabolism } \\
\text { Increased urinary phosphate excretion } \\
\text { Inhibition of osteoblastogenesis/increased RANKL }\end{array}$ \\
\hline Calcineurin inhibitors* & Ciclosporin A (high doses), tacrolimus & Increased bone turnover. Increased RANKL expression \\
\hline Parenteral nutrition & & Unclear \\
\hline
\end{tabular}

*Evidence for an association with fracture risk. SSRI, selective serotonin reuptake inhibitors; GnRH, gonadotropin-releasing hormones. 
there is a strong association between some drugs categories and fragility fractures. A significant increase in the risk of vertebral fractures [6 studies: odds ratio (OR) 1.50; 95\% confidence interval (CI) 1.32-1.72] and hip fractures (10 studies: OR 1.23; $95 \%$ CI 1.11-1.72) with proton pump inhibitors (PPIs) has been demonstrated, especially when treatment exceeds 12 months (43). An association between use of selective serotonin reuptake inhibitors (SSRIs) and hip fractures is present in the first year of treatment, for both genders, especially after the age of 70 years (44). Levothyroxine (if in excess) is associated with an increased risk of fractures in both men and women (OR 1.98; 95\% CI 1.15-1.76) (45). The use of pioglitazone and rosiglitazone is associated with a significant increase (3to 4-fold) in the risk of hip and humerus fractures in postmenopausal women (46). A large body of evidence is available on the association of some first generation anticonvulsants (carbamazepine, oxcarbazepine, phenobarbital, phenytoin, primidone) with low bone mass, as well as with a 2- to 6- fold increased risk of hip fracture in patients with epilepsy, especially when polymedicated (47). The use of cyclosporine in transplanted patients is associated with an increase in clinical fractures that ranges from $10 \%$ to $34 \%$ in the first year of treatment (48). Long-term use of unfractioned heparin increases fracture risk by $2.5 \%$ to $5 \%$, whereas there are no data on low molecular weight heparin. There is controversy in the literature about the effect of warfarin on risk of osteoporosis and fractures $(42,49)$. Some drug categories may interfere with the anti-fracture efficacy of bisphosphonates. A possible dose-dependent attenuation of the anti-fracture effect of alendronate and risedronate by PPIs has been reported in 4 epidemiological studies (two retrospective and two cohort studies), although this effect was not confirmed in a post-hoc analysis of three RCTs on risedronate (40). In a retrospective study in which alendronate was co-administered with SSRIs, there was a significant association with the risk of major osteoporotic fractures. There are no studies on the ef- fect of bisphosphonates co-administered with drugs potentially detrimental to bone health (40).

\section{Adjuvant hormonal therapy}

The substantial hypoestrogenism induced by adjuvant hormonal therapy with aromatase inhibitors or tamoxifene + LHRH analogues in women with breast cancer, and the androgen deprivation induced by $\mathrm{GnRH}$ agonists and/or antiandrogens in men with prostate cancer, lead to an accelerated bone loss and rapidly increase fracture risk (5053). There is a substantial difference in the rate of bone turnover, and thereby in the rate of bone loss, among different patient populations (men, premenopausal and postmenopausal women at diagnosis) and with different types of antihormonal therapy (chemotherapy-induced menopause, GnRH with or without tamoxifen, or aromatase inhibitors from oils, androgen deprivation therapy). Patient categories at the highest risk of osteoporosis are (in descending order): premenopausal women with chemotherapy-induced menopause treated with GnRH agonists, men on androgen deprivation, women switched from tamoxifen to aromatase inhibitors, women on aromatase inhibitors, especially if aged $<70$ years $(52,54,55)$.

Given the high prevalence of risk factors for fractures, irrespective of hormone therapy, and the high prevalence of vertebral fracture at cancer diagnosis, all subjects with breast or prostate cancer should be assessed for fragility fractures (including morphometric vertebral fractures) $(56,57)$. Aminobisphosphonates and denosumab are the first line drugs for the management of bone health in breast and prostate cancer, being able to prevent BMD loss during adjuvant hormonal therapy. The bisphosphonates alendronate, risedronate and ibandronate have been used in both men and women, at the same doses used for fracture risk reduction in postmenopausal osteoporosis $(58,59)$. Zoledronic acid has been used at doses double those used in postmenopausal osteoporosis (4 mg every 6 months) on average, with effects similar to those achieved in postmenopausal os- 
teoporosis (60). Data on the anti-fracture efficacy of bisphosphonates in this patient population are lacking. Anti-fracture efficacy has been demonstrated for denosumab $60 \mathrm{mg}$ every 6 months, both in prostate cancer patients for spine fractures and in postmenopausal women treated with aromatase inhibitors for all clinical fractures (vertebral and non-vertebral) $(61,62)$. Denosumab, at a dose of $60 \mathrm{mg}$ every 6 months, may reduce all vertebral and nonvertebral fractures by $50 \%$, and new vertebral fractures by $60 \%$. The anti-fracture effect is independent of age, duration of hormonal therapy and BMD value. In other patient populations (premenopausal women treated with GnRH agonists, women with chemotherapy-induced menopause) only the treatment effect on BMD has been evaluated. However, at the same doses used in postmenopausal or male osteoporosis, the magnitude of effect on BMD in cancer treatment-induced bone loss (CTIBL) was comparable, which suggests a similar antifracture effect (58).

There is no international consensus on the intervention threshold for the prevention of CTIBL and CTIBL-related fractures. Over time, an increasingly conservative threshold has been considered, up to nearnormal, especially in the presence of other independent risk factors $(58,59)$.

However, based on the following factors: i) the lack of evidence for a validated $\mathrm{T}$ score threshold (only based on expert opinion) and the uncertainty on the predictive value of BMD for fracture risk in this patient population; ii) a particularly fast rate of bone loss in all forms of osteoporosis induced by adjuvant hormonal therapy, as an independent risk factor; iii) a very high prevalence of osteoporosis/fractures and/or other risk factors for fracture in patients with breast or prostate cancer; iv) the strong evidence that treatment with antiresorptives is more effective when used before than after a fracture or BMD loss has occurred, in both men and women (either pre- or post-menopausal) $(60,63)$; v) the evidence that fracture risk reduction (with denosumab) is independent of BMD values at initiation of antiresorptive therapy (61).
These guidelines recommend the use of bisphosphonates or denosumab at initiation of adjuvant hormonal therapy or at the onset of chemotherapy-induced amenorrhea (www.aiom.it 2015 guidelines).

The optimal duration of bisphosphonates or denosumab therapy for osteoporosis in women with breast cancer or men with prostate cancer has not been established. For women with breast cancer and for men, it is reasonable to recommend continuing treatment through the duration of treatment with GnRH and/or aromatase inhibitors and androgen deprivation. Data supporting this recommendation are indirect $(58,64)$.

\section{OSTEOPOROSIS IN CHRONIC KIDNEY DISEASE AND ORGAN TRANSPLANTATION}

Fragility fractures are among the most frequent complications of chronic kidney disease (CKD) and in organ-transplanted patients. In recent years, a large number of epidemiological studies have shown that the incidence of hip fractures among individuals on dialysis is at least 3-fold that in the general population (Level of evidence 1) $(65,66)$. Furthermore, it is known that mortality from hip fractures in these subjects is at least twice that in the general population, as early as in the first year after the fracture event.

Furthermore, the prevalence of vertebral fractures is approximately 50\% among subjects on periodic dialysis (Level of evidence 3 ). These data are not surprising when considering the many factors that contribute, in most subjects with end-stage renal disease, to the development of renal osteodystrophy, i.e., a condition characterised by a range of bone disorders (Table IX) and known for inducing extreme bone fragility.

Epidemiological data on organ transplant are less accurate, due to the relatively small number of patients included in available studies $(67,68)$. However, it is known that the estimated prevalence of fragility fractures is approximately $10-15 \%$ among subjects waiting for solid organ transplantation (kidney, heart, liver, lung), due to 
Table IX - Renal osteodystrophy: histological classification.

\begin{tabular}{|l|l|l|}
\hline Renal osteodystrophy subtypes & Bone turnover & Bone mineralisation \\
\hline Osteitis fibrosa & High & Normal \\
\hline Osteomalacia & Normal & Low \\
\hline Adynamic bone disease & Low & Normal \\
\hline Mixed bone disease & High & Low \\
\hline
\end{tabular}

the negative effects of the underlying disease on bone. The proportion of patients with osteoporosis increases dramatically after transplantation. Bone loss is maximal in the first year after transplantation and may persist afterwards, although at a slower rate (67-69). After transplantation, more than $10 \%$ and approximately $50 \%$ of subjects with renal disease experience fragility fractures in the appendicular skeleton and vertebral fractures, respectively. Among liver, heart or lung transplanted patients, the incidence of vertebral fragility fractures is maximal in the first three years after organ transplantation, being approximately $30-40 \%$ and increasing to approximately $50 \%$ in the following years (Level of evidence 3). The main risk facture for fractures is immunosuppressive therapy, particularly glucocorticoid therapy, which is initially administered at high doses and continued for indefinite time in most patients. Other important risk factors, in all types of transplant patients, include age, female gender (in the long term). Factors specifically related to the organ disease may be crucial for the development of bone fragility. The most representative example is persistent secondary hyperparathyroidism, sometimes severe and very long term, which involves up to $50 \%$ of kidney transplanted patients, even in the presence of a well-functioning graft.

\section{Recommendations}

The use of DXA for fracture risk prediction in patients with CKD or on dialysis is not well standardized. Densitometric values by DXA can be interpreted similar to those from patients with normal kidney function only in patients with CKD stage 1-3 [glomerular filtration rate $(\mathrm{GFR}) \geq 30 \mathrm{~mL} / \mathrm{min}$ ] (Level of evidence 2). The reason for this lies in the fact that the bone disorder found in these subjects is very similar to osteoporosis of subjects with normal kidney function. Therefore, these subjects should always undergo DXA assessment to evaluate bone fragility (Grade A recommendation). In subjects wit CKD stage 4-5 and 5D i.e. with residual kidney function $\leq 29 \mathrm{ml} / \mathrm{min}$ or on dialysis), measurement of BMD does not appear to adequately predict the risk of fracture, and cannot differentiate among the different components of renal osteodystrophy (Table IX, Level of evidence 1). DXA should always be performed in organ-transplanted patients (69), immediately after transplantation and every 18 months thereafter for the first three years (Grade B recommendation). A spine X-ray should be taken every year, at least for the first 2 to 3 years following transplantation, to assess vertebral fractures (Grade $\mathrm{C}$ recommendation).

Prevention of fragility fractures, especially of vertebral fractures, is effectively achieved in subjects with CKD stage 1-3, with the same efficacy and safety as in patients with normal kidney function, using alendronate, risedronate, denosumab and teriparatide (Level of evidence 1b, Grade A recommendation). Data on the efficacy of denosumab in a very small number of subjects with CKD stage 4 are available, but do not allow drawing definite conclusions. Bisphosphonates and teriparatide have not been adequately assessed in patients with CK stage 4-5 and 5D, and are generally contraindicated in these patients.

There is insufficient evidence available from randomized, controlled, double blind studies on the anti-fracture efficacy in posttransplant osteoporosis with any drug.

Alendronate, pamidronate, ibandronate and zoledronate have been shown to in- 
crease bone mass, without causing relevant adverse events, and particularly without affecting renal function (Level of evidence 2 ). Several studies, although in a very small number of patients, have shown a prophylactic effect of intravenous ibandronate, pamidronate and zoledronic acid on vertebral fractures, with no significant adverse events and no significant changes in kidney function in kidney transplanted patients (Level of evidence 4).

With regard to the vitamin D endocrine system, it is known that progressive impairment of kidney function induces a substantial reduction in the active metabolite calcitriol, accounting for most metabolic alterations involved in the development of renal osteodystrophy (Level of evidence 1). In subjects with CKD stage 4-5 and 5D whose PTH levels are particularly high (5-fold above the upper limit of normal) and progressively increasing, calcitriol and its analogues, as well as vitamin D analogues (paracalcitol in particular) can decrease PTH levels and improve alterations of bone metabolism (Level of evidence 1a, Grade A recommendation), except for patients with adynamic bone disease. However, in recent years it has been recognized that 25-OH-vitamin D deficiency is present in nearly $90 \%$ of subjects, with or without advanced CKD (Level of evidence 2). Measurement of serum $25(\mathrm{OH})$ vitamin $D$ is highly recommended for all subjects with CKD (Grade B recommendation).

Supplementation with cholecalciferol and native vitamin D can substantially and significantly reduce PTH levels in subjects with CKD 1-5 and 5D (Level of evidence 4). Similarly, hypovitaminosis D is present in a significant proportion of organ-transplanted patients (70), up to over $80 \%$ (Level of evidence 3). Supplementation with cholecalciferol and calcidiol is strongly recommended (Grade B recommendation), with dosing regimens similar to those used in the general population (Level of evidence 3 ).

\section{DIAGNOSIS}

\section{DIAGNOSTIC PROCEDURES}

\section{Bone densitometry}

Bone mass assessed as BMD can be measured by different techniques that are generally identified as bone densitometry. Bone densitometry allows measuring bone mass rather accurately and, at present, is the best predictor of osteoporotic fracture risk.

A densitometric diagnosis of osteoporosis (71-73) is based upon the comparison between the BMD value of the subject being examined, expressed as $\mathrm{SD}$, and the mean BMD in the young normal subjects [peak bone mass; (T-score)]. BMD may also be expressed in relation to the mean BMD for subjects of the same age and gender (Z-score).

It should be noticed that, according to the WHO, the threshold for diagnosing osteoporosis (T-score $<-1.5 \mathrm{SD}$ ) is only applicable to BMD values obtained by DXA.

The WHO criteria for the diagnosis of osteoporosis are not applicable to premenopausal women nor to men younger than 50 years (Table X).

\section{Techniques for assessing bone mineral density}

At present, DXA is the preferred technique for assessing bone mass, allowing for diagnosing osteoporosis, fracture risk prediction and follow-up. It is a DXA technique that allows the assessment, virtually for each skel-

Table X - Use of T-score and Z-score in densitometry reporting.

\begin{tabular}{|l|l|}
\hline T-score & Z-score \\
\hline $\begin{array}{l}\text { In postmenopausal women and men older } \\
\text { than } 50 \text { years use the WHO BMD diagnostic } \\
\text { classification. }\end{array}$ & In premenopausal women and in men younger than 50 years. \\
\hline & $\begin{array}{l}\text { If the Z-score is -2 SD or lower, BMD is defined } \\
\text { as below the expected range for age. } \\
\text { if the Z-score is above than -2 SD BMD is defined } \\
\text { as within the expected range for age. }\end{array}$ \\
\hline
\end{tabular}

WHO, World Health Organization; BMD, bone mineral density. 
Table XI - Fracture risk predictive ability of dual-energy x-ray absorptiometry and ultrasound techniques for different sites. Values represent the increase in relative risk ( $95 \%$ confidence intervals) for every 1 standard deviation decrease (T-score).

\begin{tabular}{|l|l|l|l|l|}
\hline \multirow{2}{*}{ BMD measurement site } & \multicolumn{4}{|c|}{ Relative risk of fracture } \\
\cline { 2 - 6 } & Forearm & Hip & Vertebral & All \\
\hline DXA Proximal radius & $1.8(1.5-2.1)$ & $2.1(1.6-2.7)$ & $2.2(1.7-2.6)$ & $1.5(1.3-1.6)$ \\
\hline DXA Distal radius & $1.7(1.4-2.0)$ & $1.8(1.4-2.2)$ & $1.7(1.4-2.1)$ & $1.4(1.3-1.6)$ \\
\hline DXA Femur & $1.4(1.4-1.6)$ & $2.6(2.0-3.5)$ & $1.8(1.1-2.7)$ & $1.6(1.4-1.8)$ \\
\hline DXA Lumbar spine & $1.5(1.3-1.8)$ & $1.8(1.2-2.2)$ & $2.3(1.9-2.8)$ & $1.5(1.4-1.7)$ \\
\hline DXA Calcaneus & $1.6(1.4-1.8)$ & $2.0(1.5-2.7)$ & $2.4(1.8-3.2)$ & $1.5(1.3-1.8)$ \\
\hline Ultrasound Calcaneus & - & $2.2(1.8-2.7)$ & $1.8(1.5-2.2)$ & $1.5(1.4-1.7)$ \\
\hline Ultrasound phalanxes & - & $1.9(1.5-2.4)$ & $1.6(1.4-1.9)$ & $1.4(1.3-1.6)$ \\
\hline
\end{tabular}

BMD, bone mineral density; DXA, dual-energy $x$-ray absorptiometry.

etal site, of bone mineral content (BMC, g/ $\mathrm{cm}$ of bone segment) projected onto a bone area, to obtain a parameter defined as BMD $\left(\mathrm{g} / \mathrm{cm}^{2}\right.$ of bone segment). BMD correlates with fracture risk: for every 1 SD decrease in BMD (approximately 10\%), there is a 1.5-3 fold increase in fracture risk at any site. In general, BMD measured at one site provides a more accurate estimate of fracture risk for that site (Table XI).

As vertebral and hip fractures are the most clinically relevant osteoporotic fractures, lumbar spine and proximal femur are the most frequently assessed sites.

The accuracy of the densitometric measurement is diminished by possible interfering conditions, which should be taken into account when reporting or performing the measurement. As an example, a vertebral fracture or a vertebra with focal degenerative arthritic changes should be excluded from the densitometric analysis. However, a minimum of two lumbar vertebrae should be available for DXA reporting. Lumbar densitometric assessment is often less accurate in individuals aged over 65 years, due to the presence of arthrosic manifestations, extraskeletal calcifications or vertebral fractures. For these reasons, femoral densitometry may be preferred after this age.

Recommendations on bone mineral density measurement sites by dual-energy $x$-ray absorptiometry

Central DXA measurement is recommended for lumbar spine (L1-L4) and proximal femur:
1) Lumbar spine: i) vertebrae with structural abnormalities such as fractures, focal lesions or other abnormalities should be excluded from the analysis; ii) vertebrae should also be excluded if there is a T-score difference of more than 1.0 compared to the adjacent vertebrae; iii) a minimum of 2 lumbar vertebrae must be evaluable in order for the assessment to be considered sufficiently accurate.

2) Proximal femur: BMD of both femoral neck and total hip should be assessed for diagnostic purposes, and the lowest value should be considered.

The lowest $\mathrm{T}$-score among the 3 sites (spine, total hip or femoral neck) should be considered for densitometric classification. A diagnosis of osteoporosis shouldn't be based only on a densitometric report; it also requires a clinical evaluation.

Peripheral measurements, e.g., at the forearm, should be limited to the following situations: i) patients in whom the lumbar and/or hip assessment is not feasible or not accurate; ii) severely obese patients; iii) hyperparathyroidism.

Recently, DXA software programs that allow to assess some geometric parameters related to bone strength have been developed, such as the hip structure analysis (HAS) and the trabecular bone score (TBS). TBS is a software program that, once added to the DXA system, analyzes the level of inhomogeneity of the vertebral densitometric scan, providing indirect information on trabecular microarchitecture. Studies published so far show that TBS 
is associated with an improvement in the ability of predicting fracture risk as compared to BMD alone. This application was approved by the Food and Drug Administration (FDA), although its clinical utility has yet to be established.

\section{Quantitative computerized tomography}

Quantitative computerized tomography (QCT) allows measurement of volumetric $\mathrm{BMD}\left(\mathrm{g} / \mathrm{cm}^{3}\right)$ of the spine and hip, both total and compartmental, being able to separate trabecular BMD form cortical BMD. There is sufficient evidence supporting the predictive ability of QCT for vertebral, but not for hip, fracture risk in postmenopausal women. There is lack of sufficient evidence for fracture prediction in men. Furthermore, QCT is associated with a high amount of radiation exposure of patients (approximately $100 \mu \mathrm{Sv}$ ). At present, DXA is preferred over spine QCT for its precision, shorter scanning time, more stable calibration, lower radiation exposure and lower cost. A QCT for measuring peripheral bone sites (pQCT) is also available. Besides the measurement of total and compartmental volumetric BMD at the radius and tibia, $\mathrm{pQCT}$ allows measuring some geometric parameters related to bone strength (cortical thickness, cross-sectional area, moments of inertia, etc.). Radiation exposure for each measurement is low (approximately $5 \mu \mathrm{Sv}$ ). There is sufficient evidence for its predictive ability only for hip fractures in postmenopausal women. At present, pQCT has not a specific role in the diagnostic workup of osteoporosis. Highresolution pQCT (HR-pQCT) has high spatial resolution, therefore it can be used to assess some bone microarchitecture parameters (trabecular thickness, cortical porosity, etc.) with good accuracy. However, there is insufficient evidence on its ability to improve prediction of fragility factures, and its use is currently confined to the research setting.

\section{Quantitative ultrasound}

Quantitative ultrasound (QUS) provides two parameters (speed and attenuation) that serve as indirect indices of bone mass and structural integrity, and that are mainly measured at two sites, i.e., phalanxes and heel. It has been shown that ultrasound-derived parameters are not inferior to lumbar or hip DXA in predicting the risk of osteoporotic fractures (hip and spine), both in postmenopausal women and in men (Table XI). Bone US does not measure bone density directly. Conflicting results from US and DXA are not surprising nor infrequent, and are not necessarily indicative of an error. Rather, QUS parameters are independent predictors of fracture risk, as they are affected by other bone characteristics. As such, QUS cannot be used for diagnosing osteoporosis with the WHO criteria (Tscore $<-2.5$ ). The heterogeneity of ultrasound machines, which provide values that are not always comparable, is an important limitation of QUS. QUS may be useful when lumbar or hip DXA is not feasible, and can be recommended for epidemiological studies and first line screening, given its relatively low cost, portability and lack of radiation exposure. Therefore, when DXA cannot be performed, a low QUS value in conjunction with other clinical risk factors for fractures may justify treatment, whereas a high QUS value in the absence of risk factors suggests a low probability of osteoporotic fractures and therefore the uselessness of further investigations

\section{Follow up}

Assessments of BMD over time may be useful both for monitoring the efficacy of treatments and to identify subjects who are losing bone at a fast rate. The annual bone loss in postmenopausal women is 0.5 $2 \%$, and most treatments increase BMD by $1-6 \%$ per year. These changes should be compared with the least significant change (LSC), i.e., the minimal change detectable with the technique used that is not attributable to measurement error. The LSC may vary from 2 to $4 \%$, depending on the site and technique used, therefore a follow up measurement is generally justified after 1.5-2 years (Table XII), and never earlier than 1 year. This time interval may be shorter in specific conditions of accelerated bone loss (e.g., high dose glucocor- 
Table XII - Follow up bone mineral density testing not justified earlier than 1 year.

\begin{tabular}{|l|l|}
\hline Spine DXA & 18 months \\
\hline Hip DXA & $18-24$ months \\
\hline
\end{tabular}

DXA, dual-energy x-ray absorptiometry.

ticoid therapy, malignancy, primary and secondary hyperparathyroidism, prolonged immobility). Only densitometric measurements performed with the same instrument in quality-controlled facilities.

Densitometric lumbar spine imaging is more sensitive to longitudinal changes. As such, it should be preferred for monitoring bone mass, provided that concomitant conditions that can affect its precision are ruled out. Peripheral densitometric measurements (x-ray and ultrasound) are not recommended for follow-up, as a substantial period of time is necessary to detect significant changes in individual patients.

\section{Indications for bone mineral density} testing

Bone densitometry is recommended: i) in women aged 65 years and older, and men aged 70 years and older; ii) at any age, in

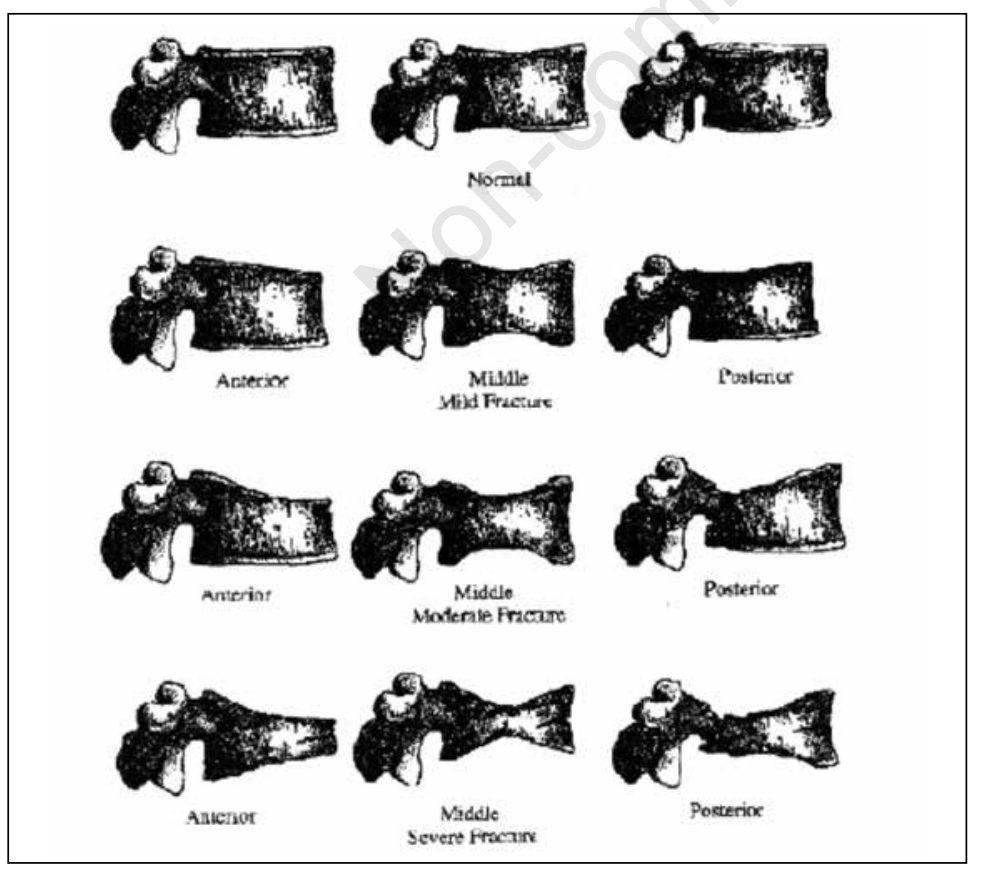

Figure 1 - Definition of vertebral fractures according to the semiquantitative method of Genant, as a $20 \%$ reduction in height of a vertebral body. subjects with previous fragility fractures, $\mathrm{x}$ ray evidence of osteoporosis or major risk factors for osteoporosis (use of drugs associated with bone loss, or conditions associated with osteoporosis); iii) postmenopausal women or men older than 60 years with risk factors (menopause before 45 years of age, or premenopausal amenorrhoea $>6$ months, inadequate calcium intake or risk factors for hypovitaminosis $\mathrm{D}$, prolonged periods of immobility, cigarette smoke, alcohol abuse, thinness, family history).

\section{Diagnosis of vertebral fractures}

Independent of BMD, a non-traumatic vertebral fracture indicates bone fragility. If due to osteoporosis, a vertebral fracture is a strong indication for pharmacological treatment aimed at reducing the risk of future fractures. As most vertebral fractures are mild in severity and asymptomatic, imaging studies are the only way to make a diagnosis.

Vertebral fractures are defined according to the semiquantitative (SQ) method of Genant, as a $20 \%$ reduction in height of a vertebral body (Figure 1) (74).

The SQ method is based on a first visual inspection of spine images for the differential diagnosis of vertebral deformities, followed by visual grading of the osteoporotic vertebral fracture as mild, moderate or severe (Genant criteria). Vertebral morphometry is a quantitative method used for diagnosing vertebral fractures by measuring vertebral heights. Vertebral morphometry should be used to assess the severity of a vertebral fracture previously diagnosed by the SQ method, and to identify new vertebral fractures during follow-up. However, vertebral morphometry should always follow a qualitative analysis of spine images to rule out causes of deformity other than osteoporosis.

Spine images can be acquired via conventional $\mathrm{x}$-ray or DXA, using the Vertebral 
Fracture Assessment (VFA) software program that a densitometer may be provided with. VFA allows acquiring a radiographic image of the entire thoracic and lumbar spine, with a low radiation exposure for patients $(50 \mu \mathrm{Sv}$, approximately $1 / 100$ of a conventional x-ray). After SQ assessment, this image is used for vertebral morphometry, i.e., the measurement of the vertebral heights. At present, DXA technology delivers high-resolution images that can be used for diagnosing and monitoring osteoporotic vertebral fractures (75-86). However, two requirements are necessary for VFA to achieve good diagnostic accuracy: i) a densitometer delivering images with good spatial resolution $(\geq 2 \mathrm{lp} / \mathrm{mm})$; ii) the operator who interprets the images should have adequate experience, documented by certified specific training, and such to differentiate vertebral fractures from deformities or other alterations.

If vertebral fractures caused by conditions other than osteoporosis are suspected, VFA should be complemented with other conventional radiology techniques or second level investigations (CT/MRI) (Table XIII).

\section{Indications for vertebral fracture testing} Conventional spine x-ray or VFA are indicated:

- in the presence of symptoms suggestive for vertebral fracture: intense back pain that worsens with standing, current or past

- in the absence of symptoms: i) in all women aged $>70$ years and men aged $>80$ years; ii) in all women aged between 65 and 69 years and in men aged between 70 and 79 years, if T-score $<-1.5$; iii) in postmenopausal women and men aged 50 years or older with specific risk factor:
- Previous fragility fractures

- A height loss $>4 \mathrm{~cm}$ in comparison with young age or $>2 \mathrm{~cm}$ from the last visit

- Marked reduction in densitometric values (T-score $<-3$ )

- Glucocorticoid therapy with prednisone $>5 \mathrm{mg}$ per day or equivalent for $>3$ months

- Comorbidities associated with an increased risk of vertebral fractures per se.

\section{Spine magnetic resonance imaging}

Use of magnetic resonance imaging (MRI) as a diagnostic tool for vertebral fragility fractures is indicated when multiple fractures are present. In fact, based on signal alterations on STIR- and T2-weighted images due to bone edema, MRI allows to differentiate recent from older fractures, and to identify vertebrae free of deformities but in which a structural failure is imminent.

\section{Spine computerized tomography}

Computerized tomography allows a detailed evaluation of the bone component of a fractured vertebra, providing useful information, e.g., on the possible dislocation of bone fragments into the spinal canal; therefore, in selected cases CT is a useful investigation that complements MRI.

\section{BIOCHEMICAL DIAGNOSIS}

An adequate evaluation is particularly recommended in patients with osteoporosis or osteopenia more severe than expected for age. Laboratory testing should be considered essential to complete the diagnostic workup of osteoporosis, as it: i) may facilitate differential diagnosis with other metabolic bone diseases that may be associated with

Table XIII - Sensitivity, specificity and levels of evidence on the clinical use of imaging techniques for vertebral fractures.

\begin{tabular}{|l|l|l|l|l|}
\hline Assessment method & Sensibility & Specificity & Diagnostic use & Follow-up \\
\hline SQ-Rx & +++ & +++ & A & A \\
\hline SQ-VFA & +++ & ++- & A & A \\
\hline X-ray morphometry & +++ & +- & B & B \\
\hline VFA morphometry & ++- & +- & B & B \\
\hline
\end{tabular}

$S Q$, semiquantitative; VFA, vertebral fracture assessment. 
clinical or densitometric features similar to those found in osteoporosis; ii) may identify potential causative factors, allowing for a diagnosis of secondary osteoporosis and, whenever possible, an aetiological therapy; iii) may guide treatment decisions and help assess treatment adherence.

Osteoporosis may be a manifestation of several diseases. In $90 \%$ of cases, normal first tier laboratory results rule out other disorders of forms of secondary osteoporosis (Grade A recommendation). For specific clinical suspicions, more targeted, second tier laboratory investigations are necessary (Table XIV).

- The choice of investigations to identify secondary forms of osteoporosis should be based on disease prevalence, clinical and drug history, and physical examination.

- Laboratory investigations to rule out secondary causes of osteoporosis should be prescribed when BMD is below the expected range for age (Z-score), or whenever satisfactory densitometric results are not achieved despite adequate therapy, in terms of persistence and compliance.

\section{Biochemical markers of bone turnover}

Biochemical markers of bone turnover can be measured in serum and/or urine, and are classified as markers of bone formation (bone-specific alkaline phosphatase, osteocalcin, propeptides of type I procollagen) and markers of bone resorption (pyridinolines, deoxypyridinolines, $\mathrm{N}$ - and $\mathrm{C}$ - terminal telopeptides of type I collagen).

In adult subjects, an increase in bone turnover markers above the normal range indicates accelerated bone loss or other primary or secondary bone disorders (e.g. nutritional osteomalacia, Paget disease, bone metastases).

In population studies, particularly in elderly postmenopausal women, bone turnover markers may be useful in estimating fracture risk (Level of evidence 2), even regardless of BMD. Bone turnover markers are overall indices of bone remodeling, and may be useful for assessing the response to therapy and treatment adherence.

Table XIV - First and second tier investigations.

\begin{tabular}{|l|}
\hline First tier investigations \\
\hline ESR \\
\hline Complete blood count \\
\hline Serum protein electrophoresis \\
\hline Serum calcium levels \\
\hline Serum phosphate levels \\
\hline Total alkaline phosphatase \\
\hline Serum creatinine \\
\hline 24-h urinary calcium \\
\hline Second tier investigations \\
\hline lonized calcium \\
\hline TSH \\
\hline PTH \\
\hline Serum 25-OH-vitamin D \\
\hline Cortisol levels after overnight dexamethasone suppression test \\
\hline Total testosterone in males \\
\hline Serum or urine immunofixation \\
\hline Anti-transglutaminase antibodies (+total lg and with free gluten-containing diet) \\
\hline Other specific investigations for associated diseases (e.g., ferritin levels, transferrin saturation, tryptase, etc.) \\
\hline
\end{tabular}

ESR, erytrocyte sedimentation rate; TSH, thyroid stimulating hormone; $\mathrm{PTH}$, parathyroid hormone. 
In this light, the advantage of using bone markers rather than densitometry lies in the shorter time needed to evaluate the efficacy of antiresorptive or anabolic therapy in the individual patient.

Use of bone markers (for estimating fracture risk and monitoring response to treatment) is complicated by the wide variability of dosing methods and biological variability, which impacts their use in individual patients. Therefore, bone markers cannot be used for routine clinical evaluations at present; their use should be limited to selected cases (e.g., an increase in total alkaline phosphatase due to liver disease, evaluation of the sustained effect of bisphosphonates after treatment discontinuation).

\section{OSTEOPOROSIS IN MEN}

Osteoporosis in men is also a public health burden. Approximately $20 \%$ of hip fractures occur in men, and the incidence of vertebral fractures in men is approximately half of that in women. However, mortality and morbidity from spine and hip fractures are higher compared with women (87). Male osteoporosis is often secondary to other causes (approximately 2/3 of cases vs $1 / 3$ of cases in women), therefore conditions associated with osteoporosis should always be ruled out (88). The most common secondary causes in men include hypogonadism, alcohol abuse, multiple myeloma, hyperparathyroidism, malabsorption, and glucocorticoid use.

In men with previous fragility fractures, a densitometric assessment is needed to confirm a diagnosis of osteoporosis. In men without fractures, measurement of bone mass by DXA is the method of choice for defining fracture risk (Level of evidence 1). BMD testing by DXA is justified in males of any age with a major risk factor (e.g., previous fragility fracture, glucocorticoid use). BMD testing is also recommended in all men between 50 and 69 years of age with 2 or more minor risk factors. The main risk factors for fracture in men are similar to those found in women, i.e., fragility fractures, family history of osteoporotic fracture, diseases or use of drugs associated with bone loss. According to the U.S. (73) and International Society for Clinical Densitometry (71) guidelines, BMD testing by DXA is cost-effective in men older than 70 years (Level of evidence 1 ).

At present, the evidence base of densitometric criteria for diagnosing osteoporosis in men is not as solid as in women. The same diagnostic cut-off as for women is applied (Level of evidence 2, Grade B recommendation).

Data on the use of the QUS in men are similar to those reported in women, but are not conclusive (Level of evidence 2). In men, as in women, the use of QUS for monitoring the response to therapy is not recommended (Level of evidence 3) (Table XV). A conventional $\mathrm{x}$-ray of the thoracic and lumbar spine should be obtained to assess prevalent vertebral deformities in men older than 50 years with a history of fragility fractures, height loss $>4 \mathrm{~cm}$ compared to the height at 20 years of age or glucocorticoid therapy (recent or current). An $\mathrm{x}$-ray should also be obtained in men aged between 70 and 79 years with spine or hip T-score is $\leq-1.5$, and in men older than 80 years with a T-score $\leq-1$ (Level of evidence 2) (71).

Table XV - Levels of evidence for the assessment of fracture risk, diagnosis and follow-up of osteoporosis in men.

\begin{tabular}{|l|l|l|l|l|}
\hline Site/technique & Vertebral fracture risk & $\begin{array}{l}\text { Non-vertebral } \\
\text { fracture risk }\end{array}$ & Treatment monitoring & $\begin{array}{l}\text { Recommendation } \\
\text { for diagnostic use }\end{array}$ \\
\hline Spine BMD/DXA & $1 \mathrm{a}$ & $1 \mathrm{a}$ & $1 \mathrm{~b}$ & $\mathrm{~A}$ \\
\hline Femoral neck BMD/DXA & $1 \mathrm{a}$ & $1 \mathrm{a}$ & $1 \mathrm{~b}$ & $\mathrm{~A}$ \\
\hline Calcaneal BMD DXA & $2 \mathrm{~b}$ & $2 \mathrm{~b}$ & $\mathrm{ND}$ & $\mathrm{B}$ \\
\hline Calcaneal US & $1 \mathrm{~b}$ & $1 \mathrm{~b}$ & 3 & $\mathrm{~B}$ \\
\hline
\end{tabular}

BMD, bone mineral density; DXA, dual-energy x-ray absorptiometry. 
National Osteoporosis Foundation (NOF) guidelines (73) recommend initiating pharmacological therapy in subjects with a vertebral (either clinical or morphometric) or hip fracture, in those with a diagnosis of osteoporosis by DXA (T-score $\leq-2.5$ at the spine or hip) and in men older than 50 years with a 10-year risk of hip fracture $\geq 3 \%$ or a 10 -year risk of any fracture $\geq 20 \%$ using FRAX® in presence of a given value of BMD.

In Italy, two oral (i.e., alendronate and risedronate) and one intravenous (i.e. zoledronic acid) amonobisphosphonates are indicated for the treatment of idiopathic or glucocorticoid-induced male osteoporosis (Grade A recommendation). Other drugs with an indication include denosumab (idiopathic male osteoporosis and iatrogenic osteoporosis in men with prostate cancer) and strontium ranelate. Furthermore, teriparatide (20 mcg s.c. per day) has a therapeutic indication for severe idiopathic osteoporosis in men (as defined by the Note 79) and in men experiencing a new spine or hip fracture during treatment with other drugs listed in the Note 79. Drugs with proven anti-fracture efficacy in men include bisphosphonates (alendronate, risedronate, zoledronic acid) and teriparatide $(38,89-92)$.

Alendronate and risedronate improve bone mass at the spine and hip, and reduce vertebral fracture risk in primary male osteoporosis and in glucocorticoid-induced osteoporosis (Level of evidence 1). There are no data on non-vertebral fractures (Level of evidence 1).

Zoledronate was shown to improve vertebral and hipbone mass, and to reduce the risk of new fractures both in idiopathic male osteoporosis and in glucocorticoid-induced osteoporosis (Level of evidence 1).

Denosumab improves BMD in men at high risk of fracture (93), and it is indicated for the treatment of bone loss in men with prostate cancer on androgen deprivation therapy (Level of evidence 1).

Strontium ranelate was shown to increase bone mass versus placebo in men (94).

Teriparatide significantly increases spine and hip BMD, and reduces moderate-to-se- vere vertebral fractures (Level of evidence 1) (92). Teriparatide for the treatment of glucocorticoid-induced osteoporosis appears to be more effective than alendronate or risedronate in increasing BMD and reducing vertebral fractures (Level of evidence 2) (38). There is no evidence of treatments able to reduce non-vertebral fracture risk in men.

The safety profile of these drugs was comparable to that reported in postmenopausal women with regard to type and incidence of adverse events (Level of evidence 1) $(95,96)$. An adequate calcium (1000-1200 mg per day) and vitamin D (cholecalciferol 8001000 IU per day) intake in combination with drug treatment is recommended.

\section{GENERAL RECOMMENDATIONS}

Osteoporosis in men is often secondary to other causes, therefore the main conditions/diseases associated with osteoporosis should be ruled out

- BMD testing is recommended in men with at least one major risk factor or in those older than 50 years with at least two minor risk factors, or in those older than 70 years even in the absence of other risk factors for fracture (Grade A recommendation).

- The densitometric threshold for diagnosing osteoporosis in men is a T-score $>-2.5 \mathrm{SD}$ compared to young normal adult males (Grade B recommendation)

- An adequate intake of calcium and vitamin D should be recommended in conjunction with drug therapy

- In Italy, drugs with an indication for male osteoporosis include alendronate, risedronate, zoledronate, denosumab and strontium ranelate (Grade A recommendation). Alendronate, risedronate and zoledronate have an indication for glucocorticoid-induced osteoporosis (Grade A recommendation). Teriparatide treatment is indicated for severe osteoporosis, and in men who experience a new spine or hip fracture after 1 year of treatment with other drugs listed in the Note 79 (Grade A recommendation). 


\section{MANAGEMENT OF OSTEOPOROSIS: NON-PHARMACOLOGICAL INTERVENTIONS}

Osteoporosis prevention consists of measures to prevent or delay the onset of osteoporosis. On the other hand, treatment of osteoporosis refers to measures to be implemented once osteoporosis has been diagnosed in subjects with or without pre-existing fractures, at increased risk of first or new fractures. Prevention is primarily based on risk factor modification. Non-pharmacological interventions (diet, physical activity, adequate calcium intake from diet) or correction of modifiable risk factors (cigarette smoke, alcohol abuse, environmental risk factors for falls) are recommended for all subjects. Non-pharmacological interventions for prevention and treatment are the same. An adequate diet, with a correct intake of vitamin D and balanced intake of proteins, carbohydrates and fats may be useful to achieve optimal peak bone mass even at a young age.

\section{NUTRITION}

\section{Calcium intake}

The average daily intake of calcium in the Italian population is insufficient, especially in the elderly. This dietary deficiency may contribute to a negative calcium balance and induce secondary hyperparathyroidism, with detrimental consequences.

The recommended dietary allowance of calcium varies depending on age and specific conditions (Table XVI) (73).

It is recommended, whenever possible, to increase calcium intake through diet. The dose of calcium supplements should be selected based on the dietary deficiency (in general, however, doses $>500-600 \mathrm{mg}$ per day are not recommended). Calcium supplementation alone was shown able to induce modest densitometric improvements in subjects with insufficient intake and postmenopausal for more than 5 years. A slight reduction in fracture risk, particularly in the elderly, has been reported with calcium supplementation has been reported, although not by all. However, the most convincing evidence for the anti-fracture efficacy is for calcium supplementation in conjunction with vitamin D. The efficacy of adequate calcium intake is, as for vitamin $\mathrm{D}$, proportional to severity and prevalence of deficiency in the treated population.

It should be noted that the risk of kidney stones might increase with calcium supplementation, whereas it is reduced by a diet rich in calcium. Furthermore, the safety profile of calcium supplements has been matter of debate due to a potential increase in cardiovascular risk.

Therefore, it is recommended to achieve an adequate calcium intake through diet, limiting the use of calcium supplements to situations where this is not feasible, and only until the daily allowance has been achieved (Level of evidence 2; Grade A recommendation).

\section{Vitamin D}

The incidence of hypovitaminosis D is large in Italy, especially with advancing age. It should be noted that only $20 \%$ of vitamin $D$ needs are met through diet; most vitamin $\mathrm{D}$ is produced endogenously in the

Table XVI - Recommended dietary allowance.

\begin{tabular}{|l|l|}
\hline Dietary allowance & mg per day \\
\hline $1-5$ years & 800 \\
\hline $6-10$ years & $800-1200$ \\
\hline $11-24$ years & $1200-1500$ \\
\hline $25-50$ years & 1000 \\
\hline Pregnancy or breastfeeding & $1200-1500$ \\
\hline Postmenopausal women on hormone replacement therapy/Men aged 50-65 years & 1000 \\
\hline Postmenopausal women not on hormone replacement therapy/Men older than 65 years & 1200 \\
\hline
\end{tabular}


skin during exposure to UVB sunrays, although this process becomes less efficient with ageing. Therefore, supplementation is often needed, particularly in the elderly. In conjunction with an adequate calcium intake, vitamin D supplementation (cholecalciferol or ergocalciferol, i.e., $\mathrm{D}_{3}$ or $\mathrm{D}_{2}$ ) in the elderly has been shown useful even for primary prevention (Level of evidence 1A; Grade A recommendation) $(97,98)$.

However, vitamin D supplementation is associated with modest densitometric effects, which are proportional to the severity of vitamin D deficiency and have been reported only at the hip.

The anti-fracture efficacy of vitamin D is modest, and has been proven for hip and non-vertebral fractures, but not for vertebral fractures. The anti-fracture effect appears to be mediated by the reported reduction in the risk of falls.

An adequate calcium and vitamin D intake is essential in all pharmacological approaches. Calcium and/or vitamin D deficiency is the most common reason for a lack of response to pharmacological therapy for osteoporosis (99).

A slight but significant reduction in mortality has also been reported with cholecalciferol or vitamin $\mathrm{D}_{3}$ in the elderly, but there is no solid evidence for extraskeletal benefits, despite a strong pathophysiological rationale.

Daily supplementation with vitamin D is the most physiological approach; however, it is also reasonable to administer vitamin $\mathrm{D}$ at equivalent weekly or monthly doses in order to improve adherence to treatment. When vitamin $\mathrm{D}$ is administered at high doses (boluses), these should not exceed $100,000 \mathrm{IU}$, as increases in bone resorp- tion markers have been reported with doses $>100,000$ IU (100), and a paradox increase in fractures and falls has been reported with boluses of 500,000 IU (101).

Vitamin $\mathrm{D}_{3}$ should be preferred over $\mathrm{D}_{2}$ to achieve adequate $25(\mathrm{OH}) \mathrm{D}$ levels more rapidly, limiting the use of the intramuscular route only to patients with severe malabsorption syndromes.

Adequate comparative evaluations of hydroxylated vitamin D metabolites (calcifediol, 1- $\alpha$ calcidiol, calcitiol) at vitamin D-equivalent doses are lacking, as well as evidence on their anti-fracture efficacy comparable to that for cholecalciferol or vitamin $\mathrm{D}_{3}$. Of note, hydroxylated vitamin D metabolites increase the risk of hypercalcaemia and hypercalciuria, which therefore should be ruled out with serial serum and urinary calcium measurement. At present, the use of hydroxylated vitamin D metabolites is rational only in patients with severe liver impairment $[25(\mathrm{OH})$ vitamin $\mathrm{D}]$ or moderate-to-severe renal impairment, $1-\alpha$-hydroxylase deficiency, severe intestinal malabsorption or hypoparathyroidism (hydroxylated metabolites). However, an adequate intake of cholecalciferol or D3 should be ensured even in these situations, given the autocrine and paracrine effects, as well as the potential extra skeletal effects of these substances.

\section{5(OH)vitamin D measurement}

Measurement of serum 25(OH)D levels is considered the best biochemical indicator of vitamin D status ( $\mathrm{nmol} / \mathrm{L}=\mathrm{ng} / \mathrm{mL} \times 2.5$ ). Table XVII shows current indications on the interpretation of 25(OH)D levels (102-104). The risk factors for hypovitaminosis D are well known, and the safe therapeutic range

Table XVII - Current indications on the interpretation of 25(OH)D levels.

\begin{tabular}{|l|l|l|}
\hline $\mathbf{n m o l} / \mathbf{L}$ & $\mathbf{n g} / \mathbf{m L}$ & Interpretation \\
\hline$<25$ & $<10$ & Severe deficiency \\
\hline $25-50$ & $10-20$ & Deficiency \\
\hline $50-75$ & $20-30$ & Insufficiency \\
\hline $75-125$ & $30-50$ & Ideal range \\
\hline $125-375$ & $50-150$ & Possible adverse events? \\
\hline$>375$ & $>150$ & Intoxication \\
\hline
\end{tabular}


for vitamin D supplementation is wide, thanks to the expected physiological mechanisms regulating vitamin $\mathrm{D}$ hydroxylation. Therefore, measurement of serum $25(\mathrm{OH})$ D levels, whose cost is considerable, is not always justified from a health cost standpoint, especially in the elderly, as hypovitaminosis $\mathrm{D}$ is highly prevalent in this population. Neither routine nor screening assessments of serum $25(\mathrm{OH}) \mathrm{D}$ are recommended, which should be limited to doubtful cases or in the presence of comorbidities that increase the risk of severe hypercalcaemia (e.g., PHPT, granulomatosis). When recommended doses ( $<4000$ IU per day) are used, measurement of serum $25(\mathrm{OH})$ for treatment monitoring is not recommended either. If deemed appropriate in doubtful cases, serum 25(OH)D levels should not be measured earlier than 4 months, i.e. the amount of time required for $25(\mathrm{OH}) \mathrm{D}$ to reach the steady state in serum.

\section{Doses for supplementation}

The dose to be used will depend on whether vitamin $\mathrm{D}$ deficiency is to be prevented or treated.

\section{Treatment of vitamin $D$ deficiency and insufficiency}

The aim of treatment of vitamin D deficiency and insufficiency it to restore $25(\mathrm{OH})$ $\mathrm{D}$ depots and normal serum levels within a short time. The total dose to be administered over few weeks may vary, depending on the severity of vitamin D deficiency and on a patient's body mass. If deficiency or insufficiency cannot be established by clinical evaluation, measurement of serum $25(\mathrm{OH})$ $\mathrm{D}$ may be considered a good indicator of vitamin D requirements (Table XVIII).

After correction of $25(\mathrm{OH}) \mathrm{D}$ deficiency, maintenance therapy should be continued in order to avoid recurrence of vitamin D deficiency or insufficiency (see prevention).
The approximate dose indicated in Table XVIII should be administered as fractioned doses over 1 to 2 months, at daily doses of 5000-7500 IU or equivalent weekly or biweekly doses.

\section{Prevention of vitamin D insufficiency}

Prevention of vitamin D insufficiency refers to the dose of vitamin $\mathrm{D}$ to recommend for subjects in whom a deficiency has already been treated, with the aim of avoiding a recurrence. Therefore, once the deficiency or insufficiency has been corrected, sometimes using massive initial doses, vitamin D supplementation should be continued at lower but regular doses over time, taking into account the persistence of clinical conditions that are risk factors for vitamin D deficiency or insufficiency.

Table XVIII presents a list of indicative criteria. Higher doses may be required in patients with poor nutritional status, advanced age, intestinal malabsorption syndromes, obesity, or taking certain drugs (e.g., anticonvulsants or glucocorticoids).

The present recommendations are based on information about the prevalence of vitamin D deficiency in Italy, and on the fact that most guidelines $(103,104)$, although not all (102), consider a level of $30 \mathrm{ng} / \mathrm{mL}$ as the optimal level to be reached. Therefore, once the desired vitamin D levels have been achieved, even with massive initial doses, vitamin D supplementation at regular doses should be continued over time.

\section{Other nutrients}

Increasing protein intake in subjects with inadequate intake reduces hip fracture risk in both genders (Level of evidence 3 ).

Adequate protein intake is essential to maintain skeletal and muscle function, but also to reduce the risk of complications following an osteoporotic fracture.

Table XVIII - Estimated therapeutic and maintenance doses relative to vitamin D deficiency status.

\begin{tabular}{|l|l|l|}
\hline Baseline $25(\mathrm{OH}) \mathrm{D}$ level or estimated vitamin D status & Total vitamin D dose for treatment & Daily maintenance dose \\
\hline$<10 \mathrm{ng} / \mathrm{mL}$ or $25 \mathrm{nmol} / \mathrm{L}$ & 600,000 & 2000 \\
\hline $10-20 \mathrm{ng} / \mathrm{mL}$ or $25-50 \mathrm{nmo} / \mathrm{L}$ & 400,000 & 1000 \\
\hline $20-30 \mathrm{ng} / \mathrm{mL}$ or $50-75 \mathrm{nmol} / \mathrm{L}$ & 100,000 & 800 \\
\hline
\end{tabular}




\section{PHYSICAL ACTIVITY}

It is known that even short periods of immobility are highly detrimental to bone mass. It is therefore important to maintain a minimum level of physical activity.

The role of an exercise program in the prevention of osteoporosis is more uncertain. The impact of such programs varies depending on frequency, duration, intensity, and age at the beginning of the program. Furthermore, the impact is specific for the weight-bearing bone site.

In young women, exaggerated exercise at competitive level may lead to hormonal and nutritional alterations that may be detrimental to bone.

Types of exercise can be generally classified into two categories: i) aerobic or impact or weight-bearing exercise (e.g., running, soccer, basketball, volleyball, baseball, racket sports, gymnastics); ii) endurance or strength training (weightlifting, body building, swimming, cycling, use of tools for static exercises).

Studies that assessed the effects of exercise on BMD are mostly case-control, nonrandomized studies, and should be evaluated according to the age of participants. In pre-pubertal subjects and young adults, only weight-bearing exercises appear to be effective (Level of evidence 2A). At present, there is insufficient evidence to recommend such exercises for neither primary, or secondary prevention. In postmenopausal women, weight-bearing exercise may prevent the expected annual bone loss of $1 \%$ (Level of evidence 1).

Epidemiological studies on the correlation between exercise and lower risk of fracture are available.

Encouraging even modest physical activity in the elderly may also contribute to reduce the risk of falls and thereby of fracture. Despite insufficient evidence on the effects on bone mass, the recommendation to perform a minimum amount of exercise (walking for more than $30 \mathrm{~min}$ utes per day, possibly in open air) appears reasonable, due to the effect of exercise on the risk of falls and on the indirect effect on vitamin $\mathrm{D}$ levels.

\section{MEASURES TO REDUCE THE RISK OF FALLS}

Most fractures, particularly hip fractures, are associated with falls. Risk factors for falls (motor disability, balance disorders, neuromuscular diseases, impaired vision, cardiovascular disease, history of falls, drugs, cognitive dysfunction) are often modifiable in the context of a multidisciplinary intervention.

Physical activity, particularly personalized exercises to improve muscle strength, balance and walking, has been shown to reduce the risk of both falls (Level of evidence 1) and fall-related traumas (Level of evidence 2A). Fall risk self-assessment tests administered in conjunction with recommendations on the prevention of falls also have a favorable impact (Level of evidence 2A). As an example, a lesser use of psychiatric drugs is associated with a reduced risk of falls. A fall prevention strategy for the elderly including adequate vitamin D intake, physical activity and education on home risks is highly recommended (Grade A recommendation).

\section{PROTECTORS}

Attenuating the impact force on the bone segment by wearing a protector is an alternative, or rather complementary strategy to reduce the risk of fracture. Protectors have been associated with conflicting outcomes, therefore their use is recommended only in selected cases (very high risk of fall).

\section{PHARMACOLOGICAL THERAPY}

\section{INTERVENTION THRESHOLD}

The aim of osteoporosis treatment is to reduce fracture risk. Non-pharmacological interventions (diet, exercise) or correction of modifiable risk factors (cigarette smoke, lifestyle) are recommended for all subjects. Conversely, a decision to use specific drugs is dependent on the risk-benefit assessment. This ratio can be easily derived from clinical trials including large groups of patients 
with similar characteristics, using simple parameters such as the Number Needed to Treat (NNT) or the Number Needed to Harm (NNH), i.e., the number of patients who need to be treated to prevent a specific event or to cause a specific adverse event. The issue is more complex for individual patients. As such, simplifications and compromises have been used that are generally considered as reasonable. Furthermore, evaluation of treatment appropriateness includes aspects of the patient's interest in terms of treatment risk-benefit balance, but also social aspects related to proper resource use. Therefore, evaluation of the appropriateness of pharmacological treatment is complex, involving factors related to the drug (evidence of efficacy, safety data, reliability in terms of feasibility of treatment and sustained adherence, cost) but also to the patient (fracture risk, comorbidities, etc.). Specifically, accurate fracture risk assessment is as important as choosing an effective treatment.

BMD T-scores have been used by the WHO to define diagnostic thresholds (osteoporosis is defined by a T-score $<-2.5$ ), and sometimes are used as intervention thresholds, e.g. $<-2.5$ for the U.S. National Osteoporosis Foundation (73).

However, use of the diagnostic threshold, as a threshold for intervention is not acceptable. Indeed, fracture risk as defined by BMD alone does not take into account other important factors that contribute to the risk of fracture regardless of BMD, e.g., age, glucocorticoid therapy, cigarette smoke, thinness, etc., which are generally known as clinical risk factors.

Risk factors, such as history of previous osteoporotic fractures or glucocorticoid therapy at a daily dose $>5 \mathrm{mg}$ prednisone or equivalent, are associated with such a high risk of fracture that a decision to initiate pharmacological therapy may be independent of densitometric values. This has been recognized by the AIFA Note 79 , which defines the criteria for drug reimbursement in Italy.

In other situations, the estimate of risk and therefore of the intervention threshold should be based on both BMD and other clinical risk factors for fracture. An integrated assessment of multiple risk factors, possibly using a mathematical algorithm, can be expressed as lifetime risk or, more conveniently, as 10-year fracture risk (10YFR). The FRAX® tool is one of the most used algorithms, available online at https://www.shef.ac.uk/FRAX/. However, the FRAX ${ }^{\circledR}$ has intrinsic limitations mainly related to the inaccuracy of dichotomous variables such as previous fracture, the possibility to omit BMD and the exclusion of many comorbidities and other important variables.

In order to overcome the limitations of the FRAX® tool and improve its accuracy, an alternative version has been derived from FRAX®, i.e., the derived fracture risk assessment or DeFRA ${ }^{\circledR}$ (available online at: https://defra-osteoporosi.it/) that provides a risk estimate essentially similar to FRAX®, exclusively based on continuous variables (age, BMI, BMD) but more accurate in the evaluation of other clinical risk factors, this being more detailed (e.g., site and number of previous fractures) and complete (e.g., other drugs associated with bone loss, other comorbidities, vertebral BMD in addition to hip BMD) (105-107).

The DeFRA ${ }^{\circledR}$ tool allows for a rational and consistent diagnostic and therapeutic approach to osteoporosis, specifically adapted to the Italian setting and the criteria for treatment decisions defined by the AIFA. Furthermore, DeFRA allows stratification of facture risk, which can be used for choosing the most appropriate therapy in conjunction with criteria such as safety $(95,96)$, cost-effectiveness ratio (108), expected adherence and pathophysiological rationale.

However, it is not advised, at present, to make decisions on pharmacological treatment exclusively on the basis of DeFRA $\AA$ scores. DeFRA ${ }^{\circledR}$ should be considered as a tool in continuous evolution. In fact, both the algorithm and the factorial components of the individual clinical risk factors might be modified underway, based on data obtained from the DeFRA ${ }^{\circledR}$ itself or from new studies or meta-analyses in the scientific literature. 


\section{BISPHOSPHONATES}

Bisphosphonates are synthetic compounds that selectively bind to bone areas undergoing remodeling. At these sites, bisphosphonates inhibit osteoclast activity through a mechanism of action that differs depending on whether or not they contain an aminic group. All bisphosphonates developed so far for the treatment of either focal bone disorders or osteoporosis reduce bone turnover in a dose-dependent manner, with proportional increases in bone density. The intestinal absorption of bisphosphonates is only $0.5-5 \%$. Bisphosphonates currently approved for the treatment of osteoporosis in Europe include: etidronate, clodronate, alendronate, risedronate, ibandronate, and zoledronate $(72,73)$.

Etidronate and clodronate are non-amino bisphosphonates that increase vertebral BMD and maintain femoral neck BMD in postmenopausal women (Level of evidence 1). Oral clodronate at a dose of $800 \mathrm{mg}$ per day was shown effective in reducing clinical fractures. In Italy, $100 \mathrm{mg}$ per week administered via intramuscular (i.m.) route is the most commonly used dosage. Assuming an intestinal clodronate absorption of $2 \%$, the i.m. dosage would be equivalent to the oral dose with proven anti-fracture efficacy. However, studies confirming these pharmacokinetic premises by comparing the effects of oral and i.m. formulations on BMD improvements and bone turnover reduction are lacking. The recommended and currently used dose of etidronate has always been suboptimal in order to avoid defective bone mineralization. Etidronate and clodronate are therefore considered as second-line drugs that are especially used in primary prevention, given their low cost. Alendronate and risedronate may increase vertebral BMD by 10 and $6 \%$ over 3 years, respectively. Both drugs have high evidence of efficacy for the prevention of both vertebral and non-vertebral fractures (including hip fractures), which are reduced by approximately $40-50 \%$ over 3 years. Antifracture efficacy has been demonstrated for daily dosing, and can be assumed for the intermittent weekly or monthly regimens, based on equivalence of different formulations in inducing an increase in BMD. An alendronate oral solution formulation has recently become available in Italy. Recent data indicate that concomitant use of PPIs with oral bisphosphonates may reduce anti-fracture efficacy of the latter (109).

Ibandronate was approved based on studies that used the $2.5 \mathrm{mg}$ per day regimen. At this dose, ibandronate is only effective in reducing vertebral fracture risk. However, ibandronate has subsequently been marketed at a dose of $150 \mathrm{mg}$ every month or 3 mg i.v. every 3 months, i.e., at cumulativebioavailable doses twice the dose used in registration studies. When compared to the oral $2.5 \mathrm{mg}$ per day regimen, this dosage was shown able to reduce the risk of nonvertebral fractures.

Zoledronate (5 mg i.v. every 12 months) was approved for the treatment of osteoporosis based on a single study that clearly demonstrated an effect on the risk of vertebral, non-vertebral and hip fractures after 3 years of treatment. The 9-year extension study demonstrated that hip bone mass remains stable with continued treatment, although there were no significant differences in terms of BMD, bone turnover markers and incidence of new fractures as compared to the group that discontinued treatment after 6 years (110). In an ancillary study, zoledronate reduced the risk of new clinical fractures when administered 2 weeks after a hip fracture. Furthermore, a reduction of overall mortality was demonstrated for the first time with zoledronate.

Alendronate, risedronate and zoledronate are also approved for the treatment of osteoporosis in men. In a recent controlled study, zoledronate significantly reduced the risk of morphometric vertebral fractures in a large group of men with primary osteoporosis, with or without hypogonadism (111). Neridronate is the only bisphosphonate indicated for the treatment of osteogenesis imperfecta. It appears reasonable to assimilate any form of juvenile-onset idiopathic osteoporosis to osteogenesis imperfecta without performing costly and limitedly accessible genetic testing. In Italy, neridronate is also indicated for the treatment of 
algodystrophy (complex regional pain syndrome type I), based on data from a recent randomized controlled trial (112).

\section{Safety profile of bisphosphonates}

Overall, bisphosphonates have a favourable tolerability profile.

- Gastrointestinal tolerability: oral aminobisphosphonates (except for clodronate and etidronato) may cause severe oesophageal erosions. The availability of weekly and monthly formulations has led to an improvement in gastrointestinal tolerability, provided that the drug is taken correctly.

- Renal tolerability: bisphosphonates, either for oral or intravenous administration, are not associated with significant risk for renal function. However, adequate fluid intake should be ensured with intravenous bisphosphonates, and the recommended doses and infusion rates should be respected. If indicated, intravenous bisphosphonates may be used even in patients with moderate renal impairment, adjusting the dose if needed. However, intravenous zoledronate is contraindicated in patients with glomerular filtration rate less than $35 \mathrm{ml} /$ min (113).

- Acute phase reaction: Intravenous administration of amino-bisphosphonates (but also of high-dose oral bisphosphonates) may be associated with flu-like clinical manifestations of 1-3 days of duration, characterized by fever and generalized muscle aches and joint pain. Such manifestations are more frequent and severe after the first drug administration. Glucocorticoid therapy for 2-3 days may be recommended for rare cases when the symptoms are more severe and of longer duration.

- Osteonecrosis of the jaw: in the treatment of malignancies (bone metastases, malignant hypercalcaemia, etc.) bisphosphonate therapy at doses tens of times greater than those used for osteoporosis treatment is associated with an increased risk (up to $1 \%$ ) of osteonecrosis of the jaw (ONJ). Actually, it has been revealed to be an osteomyelitis almost invariably associated with an Actinomyces infection. This adverse effect of bisphosphonate therapy is reported much less frequently in patients treated for osteo- porosis, with an increase in risk following oral invasive procedures involving bone exposure. It is widely accepted that the best way of managing $\mathrm{ONJ}$ is prevention, which is essentially based on risk factor control. In patients starting bisphosphonate therapy for osteoporosis, a dental examination with dental extraction of abscessed or infected teeth is not required prior to initiation of therapy. Patients should be encouraged to maintain routine oral hygiene, similar to the general population, especially when oral hygiene is insufficient. If invasive dental procedures are necessary, initiation of therapy may be delayed until resolution of the dental problem. Alternatively, procedures can be performed within the first 6 months of therapy. In subjects treated with bisphosphonates for osteoporosis for less than 3 years and without individual risk factors (diabetes, immunosuppression, glucocorticoids, cigarette smoke, alcohol) the risk of ONJ associated with invasive procedures is extremely low, and no specific measures or precautions are required. Based on epidemiological data, a dentist's refusal to perform dental procedures, even when invasive (dental extractions), or considering bisphosphonate therapy and the dental procedure as mutually exclusive in the absence of other documented risk factors does not appear to be justified. In some cases, not performing the procedure could be a risk factor for ONJ per se. In subjects treated with bisphosphonates for more than 3 years (with compliance $>80 \%$ ), regular professional oral hygiene is recommended, in the same manner as in the general population. If oral surgery (dental extraction) is required, many guidelines recommend discontinuation of bisphosphonates for 3 months and re-initiation of therapy once the surgical wound has healed. There is no evidence that this can really reduce the risk of ONJ, considering the persistence of effect of bisphosphonates. Furthermore, for the same reason, it is likely that bisphosphonate discontinuation for a relatively short period (1-2 months) does not compromise the efficacy of treatment for osteoporosis. Recently, some authors have proposed temporarily suspending treatment follow- 
ing dental extraction, until healing of the mucosa at the site of extraction. Prolonged interruptions of treatment should be agreed upon by the dentist and the physician who has prescribed bisphosphonate therapy. In the case of invasive dental procedures (dental extraction), especially if individual risk factors (diabetes, immunosuppression, glucocorticoids, cigarette smoke, alcohol) are present, adequate antibiotic prophylaxis (amoxicillin, possibly combined with metronidazole, to be initiated 5 to 2 days prior to surgery and to be continued until healing of the mucosa) is recommended. Antibiotic prophylaxis should be prescribed for surgical dental extractions that involve primary closure of the site of extraction with mobilization of mucoperiosteal flaps. There are no contraindications to dental implant surgery during bisphosphonate treatment. Only 12 cases of ONJ associated with implant surgery are reported in the literature, with an estimated risk of implant failure of $0.88 \%$. However, peri-implantitis, a potential complication of implant surgery, can increase the risk of ONJ during bisphosphonate therapy per se. Therefore, it is essential that patients adhere to a strict oral hygiene program. The Italian Ministry of Health issued recommendations for the prevention of ONJ limited to cancer patients. Recently, a joint document on ONJ associated with bisphosphonate use both in cancer and osteoporosis was issued by the Italian Society of Maxillofacial Surgery (SICMF) and the Italian Society of Oral Diseases and Medicine (SIPMO) (114). It should be noted that many of the recommendations derived from international literature and adopted by may guidelines have a relatively low level of evidence, but a relatively good strength of recommendation based on expert opinion.

- Atypical sub-trochanteric fractures: in patients treated with bisphosphonates for many years (but also in patients never treated with bisphosphonates), occurrence of atypical sub-trochanteric femoral fractures (transverse fractures). These are stress fractures that should meet strict classification criteria for a diagnosis of atypical fracture (115). Incidence of these fractures is very low (3.2 to 50 cases per 100,000 person/ year), but clearly associated with duration of treatment. Based on available data and the rarity of these events, the risk-benefit ratio for the use of bisphosphonates in the prevention of fragility fractures is clearly in favor of a benefit. In order to minimize the risk of sub-trochanteric fracture in patients on bisphosphonate treatment, the following may be useful: i) considering periods of drug holiday after assessing the risk-benefit ratio and ii) correct and monitor other risk factors for atypical fractures (long-term glucocorticoid use, hypovitaminosis D, long-term use of PPIs, bone disorders other than osteoporosis).

For a detailed review of the safety profile of bisphosphonate, see the recently published SIOMMMS position paper (95) and available from the Society's website (www. siommms.it).

\section{Duration of therapy}

Optimal duration of bisphosphonate therapy for osteoporosis has not been established. The effects of zoledronate and alendronate on BMD and bone turnover persist for few months after discontinuation of treatment. The need for continued treatment should be periodically reassessed in each individual patient, taking into consideration benefits and potential risks of therapy, especially after 5 or more years of use. It appears reasonable to recommend discontinuing treatment for 12-24 month in patients who have been treated with bisphosphonates for more than 5 years and at low risk of fracture. It is recommended to continue treatment up to 10 years (maximal duration of treatment assessed so far) in patients at high risk of fracture, such as those with a hip T-score $<2.5$ or with previous vertebral fractures and a hip T-score $<-2$.

\section{DENOSUMAB}

Denosumab is a humanized monoclonal antibody that neutralizes RANKL, a cytokine that, by interacting with its receptor RANK on the membrane of pre-osteoclasts and mature osteoclasts, inhibits osteoclast recruitment, maturation and survival. Sub- 
cutaneous administration of denosumab leads to nearly complete inhibition of osteoclastic bone resorption, and subsequently of bone formation. As bisphosphonates, denosumab is therefore an antiresorptive drug. The main differences between these drugs are: i) the effect of denosumab ceases immediately as the drug is cleared from the plasma; therefore, if treatment is interrupted when a patient is still at increased risk of fracture, initiation of an alternative treatment should be considered to prevent rapid resumption of bone loss; ii) the consistent effect of denosumab at all bone structures, regardless of bone turnover, which translates into a greater pharmacological activity at the cortical bone; for this reason, increases in BMD are greater as compared with the most potent bisphosphonates, particularly at cortical bone structures; iii) long-term denosumab therapy is associated with sustained increase in BMD, whereas with other anti-resorptive a plateau is seen after 3-4 years of treatment, particularly at cortical bone structures.

Registration studies were conducted using denosumab $60 \mathrm{mg}$ s.c. every 6 months $(72,73)$. This dose ensures nearly complete inhibition of bone turnover, although after 2-4 years this tends to return to pre-treatment levels towards the end of the sixth month of the dosing interval. In postmenopausal women, the anti-fracture efficacy of denosumab has been documented for vertebral fractures $(-68 \%$ over 3 years of therapy), hip fractures $(-40 \%$ over 3 years of therapy) and non-vertebral fractures $(-20 \%$ over 3 years of therapy). Denosumab has also evidence of efficacy for the treatment of men at increased risk of fracture, women with breast cancer treated with aromatase inhibitors and men on androgen deprivation therapy for prostate cancer. An even greater densitometric benefit has been seen in patients with severe osteoporosis treated with either concomitant teriparatide and denosumab therapy or teriparatide following denosumab (but not vice versa).

No significant adverse events were shown in clinical trials $(95,96)$. Denosumab treatment may lead to hypocalcaemia, therefore potential risk factors for hypocalcaemia should be corrected prior to initiation of therapy. In post-registration extension studies, rare cases of ONJ have been reported. This led to considering ONJ pathogenesis as related to the reduction in bone turnover. For the same reason, use of denosumab has been associated, although rarely, with atypical femoral fractures (see bisphosphonates). A trend for a greater incidence of infections, predominantly skin infections, was seen during clinical trials. Although this was not considered as crucial by the European Medicines Agency (EMA) and FDA, continued risk assessment through the pharmacovigilance program was deemed necessary.

\section{TERIPARATIDE}

Daily subcutaneous administration of PTH, and specifically of its active 1-34 fragment (teriparatide) stimulates both bone formation and resorption, with a predominant effect on bone formation (anabolic window) that is particularly evident during the first 12 months of treatment. Observed BMD increases are significantly greater than those achieved with bisphosphonates only in trabecular bone, with an increase in vertebral BMD of nearly $10 \%$ at 18 months. However, teriparatide treatment also induces improvements in certain geometric characteristics of cortical bone that relate to its resistance to fracture.

Teriparatide was shown to reduce (after 21 months of treatment) vertebral fractures by $65 \%$ and non-vertebral fractures by 53\% $(72,73)$. A rapid decrease in BMD is observed at treatment discontinuation. It is therefore advisable to promptly initiate an alternative (anti-resorptive) treatment. The association of teriparatide with other drugs has been evaluated in several studies. So far, the greatest effect on BMD has been achieved with teriparatide in conjunction with zoledronate or denosumab. It is believed that such associations could be considered for patients at very high risk, such as those with multiple vertebral fractures or a femoral fracture. The drug is also approved for the treatment of glucocorticoidinduced osteoporosis based on a study that 
demonstrated superior efficacy of teriparatide compared to alendronate therapy, both with regard to BMD and clinical fractures. Due to its high cost, the use of teriparatide is limited to secondary prevention in patients with severe osteoporosis at increased risk of fracture or non-responsive to antiresorptive drugs. Teriparatide therapy is often associated with adverse events of mild severity (nausea, cramps in the lower limbs) and with increased incidence of hypercalcaemia, which is usually asymptomatic $(95,96)$. According to its Summary of Product Characteristics, teriparatide treatment should not exceed 24 months and should not be repeated over a patient's lifetime. This limitation poses obvious management problems. As an example, should a patient who discontinues treatment after a short period of time for reasons unrelated to drug tolerability not be treated for the rest of his life? The present guidelines consider the recommendation in the Summary of Product Characteristics as referring to a total duration of treatment of 24 months, with daily administration.

\section{STRONTIUM RANELATE}

Treatment with strontium ranelate effectively reduces vertebral, non-vertebral and hip fractures in women with postmenopausal osteoporosis. Strontium is the active ingredient of the compound, which adsorbs to hydroxyapatite crystals with labile bond. The mechanism of action of strontium ranelate is related to its interaction with the calcium sensing receptor (CaSR), and involves the OPG-RANKL system. Strontium ranelate was assessed in two 5-year clinical trials, involving over 7000 women, with the main analysis at 3 years $(72,73)$. Results at 3 years showed that strontium ranelate reduced the risk of vertebral, nonvertebral and hip fractures (in a subgroup at high risk) by 41,16 and $36 \%$, respectively. Results at 5 years confirmed the findings of the first 3 years. Strontium ranelate modestly increases markers of bone formation (approximately by $15 \%$ ), while reducing markers of bone resorption (10-15\%). The greater weight of strontium accounts for approximately $50 \%$ of the increase in BMD observed with treatment. Recently, a direct correlation between BMD and the reduction in risk for vertebral and hip fractures has been demonstrated.

Strontium ranelate therapy is associated with modest bowel movement pattern changes and a mild increase in the risk for thromboembolic events, particularly in elderly patients: the drug is contraindicated in patients with current or past venous thromboembolism (VTE) and in the case of temporary or permanent immobilization. Furthermore, the need for continued treatment with strontium ranelate should be re-evaluated in patients aged over 80 years at risk of VTE. Treatment with strontium ranelate was also associated with an increase in the risk of myocardial infarction [relative risk vs placebo $1.6(95 \% \mathrm{CI}=1.07 ; 2.38)](95$, 96). The drug is therefore contraindicated in patients with established, current or past history of ischemic heart disease, peripheral arterial disease and/or cerebrovascular disease, and uncontrolled hypertension.

Severe allergic cutaneous reactions, sometimes associated with potentially lifethreatening systemic symptoms [drug rash with eosinophilia and systemic symptoms (DRESS), Stevens-Johnson syndrome, toxic epidermal necrolysis] have been reported very rarely with strontium ranelate. In such cases, treatment should be immediately discontinued, and not re-started at any time (EMA/185175/2012). Use of strontium ranelate has been restricted to the treatment of severe osteoporosis in postmenopausal women or in adult men at high risk of fracture, for whom treatment with other medicinal products approved for the treatment of osteoporosis is not possible.

\section{HORMONE REPLACEMENT THERAPY}

Treatment with estrogens, alone or in combination with progestogens (hormone replacement therapy, HRT) or tibolone may increase bone mass. The Women's Health Initiative (WHI) study demonstrated that treatment with conjugated estrogens reduces the risk of any osteoporotic fracture. 
The favorable effect on fracture, which is accompanied by a reduction in the risk of colorectal cancer, is counterbalanced by an increased risk of breast cancer, stroke, ischemic heart disease and thromboembolic events, with an unfavorable risk-benefit ratio, particularly for long-term treatments and when combination therapy with progestogens is required (women without hysterectomy) (72, 73). Based on these data, estrogen or combined estrogen/progestogen therapy is not indicated for the treatment or prevention of osteoporosis. In women with menopausal symptoms, especially if aged $<50-55$ years, temporary (1-3 years) estrogen or combined estrogen/progestogen therapy (depending on whether or not the patient underwent hysterectomy), may be considered as somehow physiological and therefore acceptable, also for the prevention of osteoporosis.

\section{SELECTIVE ESTROGEN RECEPTOR MODULATORS}

Selective estrogen receptor modulators (SERMs) are synthetic compounds that bind the ER thereby inducing agonistic effects in bone and liver, and antagonistic effects in the mammary gland and genitourinary tract.

SERMs currently approved for the prevention and treatment of osteoporosis in Italy include raloxifene and bazedoxifene.

Raloxifene prevents bone loss in the first years after menopause, inducing a 2-3\% increase in bone density in osteoporotic women. The anti-fracture efficacy of raloxifene was assessed in a single, large study (MORE). After 3 years, raloxifene $60 \mathrm{mg}$ per day reduced the incidence of new vertebral fractures (but not non-vertebral fractures) in both women with previous vertebral fractures $(30 \%)$ and women with no previous fractures $(50 \%)$. In MORE, a significant reduction in the incidence of invasive breast cancer was also observed $(72,73)$.

Raloxifene does not improve, and may even increase the incidence of menopauseassociated vasomotor symptoms. Most commonly reported adverse events are increased vasomotor symptoms and cramps of the lower limbs. Raloxifene, as HRT, is associated with increased risk of thromboembolic events, therefore it is not recommended in patients with previous or at risk for venous thrombosis $(95,96)$.

Bazedoxifene is a third-generation SERM that, at a dose of $20 \mathrm{mg}$ per day, was shown to prevent bone loss both in normal and osteopenic women. In osteoporotic women, bazedoxifene significantly reduced the risk of fracture by $42 \%$. The 5 -year extension study showed persistence of effect on vertebral fractures (32\% reduction in risk). A post-hoc analysis in patients at high risk demonstrated a significant reduction in non-vertebral fractures both at 3 and 5 years $(72,73)$. Furthermore, bazedoxifene showed a greater anti-estrogen effect on the uterus, in the absence of significant adverse events $(95,96)$. A new strategy for ER modulation, known as tissue selective estrogen complex (TSEC), combines estrogen therapy (conjugated equine estrogens) with bazedoxifene. Data from clinical trials indicate that this association leads to improvements of menopausal symptoms and bone mass, without causing adverse effects on the breast and uterus (116-120). However, the anti-fracture and cardiovascular effects of this association have not been adequately investigated yet.

\section{NEW TREATMENT PERSPECTIVES}

Cathepsin $\mathrm{K}$ is a key enzyme for osteoclast activity, and it is therefore a potential therapeutic target. Odanacatib, a selective inhibitor of cathepsin $\mathrm{K}$, was shown able to reduce bone resorption by $50 \%$, without affecting bone formation significantly, thereby significantly increasing BMD and reducing osteoporotic fractures.

Pharmacological inhibition of sclerostin, a well-known, natural occurring inhibitor of the Wnt system, which is essential for bone formation is another potential new approach for the treatment of osteoporosis. In fact, administration of sclerostinneutralising monoclonal antibodies significantly increased BMD and bone strength in a relatively short time in preliminary experiments. 


\section{VERTEBROPLASTY} AND KYPHOPLASTY

Vertebral fractures often cause sudden and rapidly progressive pain, unrelated to traumas, that is initially continuous, also present at rest, and subsequently only occurring with load. The management of patients with acute vertebral fractures includes conservative measures such as rest, braces or corsets, minor and major analgesics. Vertebral fracture pain usually attenuates after 1-3 weeks, and abates over few months. However, in many cases pain may persist for months, depending on severity and fracture site, which impact the clinical course and may determine persistent instability (pseudoarthrosis).

Trans-peduncular injection of cement in the fractured vertebral body may induce immediate resolution of pain.

Currently available techniques for stabilizing or reducing-stabilizing vertebral fractures include vertebroplasty, where cement is injected at high pressure, with greater risk of extravasation and pulmonary embolism, and kyphoplasty, where cement is injected at low pressure, with lower risk of extravasation; theoretically, the latter technique allows reducing vertebral deformity by inserting a balloon that is subsequently inflated within the vertebral body.

Given the risks and the uncertain long-term benefits associated with these procedures, vertebroplasty or kyphoplasty should only be recommended in patients with intractable pain lasting for weeks. Both techniques should be offered for vertebral fractures causing pain for weeks after onset, whereas kyphoplasty should be proposed when re- storing the morphology of a fractured vertebral body is deemed feasible, when reduction in chest volume may impair a patient's vital capacity, or when there is a risk of a domino effect due to biomechanical imbalances.

Of course, using these procedures in patients with few or no symptoms is unadvisable.

Appropriate pharmacological treatment is essential in all patients with vertebral fragility fractures treated by vertebroplasty or kyphoplasty, in order prevent an increase in risk of adjacent vertebral fracture associated with the presence of cement in a vertebral body in a situation of generalized bone fragility.

\section{SYNOPTIC TABLES OF} PHARMACOLOGICAL THERAPY

\section{POSTMENOPAUSAL OSTEOPOROSIS}

The pharmacological therapy for postmenopausal osteoporosis is summarized in Tables XIX and XX.

\section{OSTEOPOROSIS IN MEN}

The pharmacological therapy for osteoporosis in men is summarized in Table XXI.

\section{GLUCOCORTICOID-INDUCED OSTEOPOROSIS}

The pharmacological therapy for glucocorticoid-induced osteoporosis is summarized in Table XXII.

Acknowledgments: the authors thank Luca Giacomelli, Ph.D, who provided editorial assistance.

Table XIX - Levels of evidence.

\begin{tabular}{|l|l|l|l|l|}
\hline \multirow{2}{*}{ Bisphosphonates } & \multicolumn{4}{l}{ Treatment target } \\
\cline { 2 - 6 } & BMD & Vert Fx & Non-vert. Fx & Hip Fx \\
\hline Alendronate & 1 & 1 & 1 & 1 \\
\hline Clodronate $800 \mathrm{mg} /$ die/os & 1 & 1 & 1 & - \\
\hline Etidronato & 1 & 1 & - & - \\
\hline Ibandronate & 1 & 1 & $1^{*}$ & - \\
\hline Risedronate & 1 & 1 & 1 & 1 \\
\hline Zoledronate & 1 & 1 & 1 & 1 \\
\hline
\end{tabular}

BMD, bone mineral density. *Evidence from post-hoc studies or meta-analyses (see text). 
Table XX - Levels of evidence.

\begin{tabular}{|l|l|l|l|l|}
\hline \multirow{2}{*}{ Other therapies } & \multicolumn{5}{|c|}{ Treatment target } \\
\cline { 2 - 6 } & BMD & Vert Fx & Non-vert. Fx & Hip Fx \\
\hline Teriparatide & 1 & 1 & 1 & - \\
\hline PTH 1-84 & 1 & 1 & - & - \\
\hline Strontium ranelate & $1^{*}$ & 1 & 1 & $1^{\circ}$ \\
\hline Hormone replacement therapy\# & 1 & 1 & 1 & 1 \\
\hline Raloxifene & 1 & 1 & - & - \\
\hline Bazedoxifene & 1 & 1 & - & - \\
\hline Denosumab & 1 & 1 & 1 & 1 \\
\hline
\end{tabular}

BMD, bone mineral density; PTH, parathyroid hormone. *Affected by strontium ranelate's higher molecular weight; ' ${ }^{\circ}$ evidence from post-hoc studies (see text); \#no longer indicated for the treatment of osteoporosis due to its adverse effects.

Table XXI - Levels of evidence.

\begin{tabular}{|l|l|l|l|l|}
\hline \multirow{2}{*}{ Pharmacological intervention } & \multicolumn{5}{|c|}{ Treatment target } \\
\cline { 2 - 6 } & BMD & Vert Fx & Non-vert. Fx & Hip Fx \\
\hline Alendronate & 1 & 2 & - & - \\
\hline Risedronate & 1 & 2 & - & - \\
\hline Zoledronate & 1 & 1 & 2 & 2 \\
\hline Teriparatide & 1 & 1 & - & - \\
\hline Strontium ranelate & 1 & - & - & - \\
\hline Denosumab & 1 & 1 & - & - \\
\hline
\end{tabular}

$\mathrm{BMD}$, bone mineral density.

Table XXII - Levels of evidence.

\begin{tabular}{|l|l|l|l|l|}
\hline \multirow{2}{*}{ Pharmacological intervention } & \multicolumn{5}{|c|}{ Treatment target } \\
\cline { 2 - 5 } & BMD & Vert Fx & Non-vert. Fx & Hip Fx \\
\hline Alendronate & 1 & $1^{*}$ & - & - \\
\hline Risedronate & 1 & $1^{* \circ}$ & - & - \\
\hline Clodronate & $1^{\#}$ & $2^{\#}$ & - & - \\
\hline Teriparatide & 1 & 1 & - & - \\
\hline Zoledronate & $1^{\S}$ & - & - & - \\
\hline Denosumab & $2^{\#}$ & - & - & - \\
\hline
\end{tabular}

$\mathrm{BMD}$, bone mineral density. *Non primary end-point; ${ }^{\circ}$ from a meta-analysis of 2 trials; "randomized, open label, single centre study, using $100 \mathrm{mg}$ i.m. per week; no specific therapeutic indication in the Summary of Product Characteristics; §greater densitometric increases compared to risedronate in a head-to-head study.

\section{REFERENCES}

1. Hernlund E, Svedbom A, Ivergård M, et al. Osteoporosis in the European Union: medical management, epidemiology and economic burden. A report prepared in collaboration with the International Osteoporosis Foundation (IOF) and the European Federation of Pharmaceutical Industry Associations (EFPIA). Arch Osteoporos. 2013; 8: 136.

2. Kanis JA, Hans D, Cooper C, et al. Interpretation and use of FRAX in clinical practice. Osteoporos Int. 2011; 22: 2395-411.
3. Morin SN, Lix LM, Leslie WD. The importance of previous fracture site on osteoporosis diagnosis and incident fractures in women. J Bone Miner Res. 2014; 29: 1675-80.

4. Hodsman AB, Leslie WD, Tsang JF, Gamble GD. 10-year probability of recurrent fractures following wrist and other osteoporotic fractures in a large clinical cohort: an analysis from the Manitoba Bone Density Program. Arch Intern Med. 2008; 168: 2261-7.

5. Gehlbach S, Saag KG, Adachi JD, et al. Previous fractures at multiple sites increase the risk 
for subsequent fractures: The global longitudinal study of osteoporosis in women. J Bone Miner Res. 2012; 27: 645-53.

6. Kanis JA, Johansson H, Oden A, et al. A family history of fracture and fracture risk: a meta-analysis. Bone. 2004; 35: 1029-37.

7. Hippisley-Cox J, Coupland C. Derivation and validation of updated QFracture algorithm to predict risk of osteoporotic fracture in primary care in the United Kingdom: prospective open cohort study. BMJ. 2012; 344: e3427.

8. Vestergaard P. Discrepancies in bone mineral density and fracture risk in patients with type 1 and type 2 diabetes-a meta-analysis. Osteoporos Int. 2007; 18: 427-44.

9. Janghorbani M, Van Dam RM, Willett WC, Hu FB. Systematic review of type 1 and type 2 diabetes mellitus and risk of fracture. Am J Epidemiol 2007; 166: 495-505.

10. Fan Y, Wei F, Lang Y, Liu Y. Diabetes mellitus and risk of hip fractures: a meta-analysis. Osteoporos Int. 2016; 27: 219-28.

11. van den Bos F, Speelman AD, Samson M, et al. Parkinson's disease and osteoporosis. Age Ageing. 2013; 42: 156-62.

12. Dennison EM, Compston JE, Flahive J, et al. Effect of co-morbidities on fracture risk: findings from the global longitudinal study of osteoporosis in women (GLOW). Bone. 2012; 50: 1288-93.

13. Broy SB, Tanner SB; FRAX(®) Position Development Conference Members. Official positions for FRAX® clinical regarding rheumatoid arthritis: from joint official positions development conference of the international society for clinical densitometry and international osteoporosis foundation on FRAX®. J Clin Densitom. 2011; 14: 184-9.

14. Shiau S, Broun EC, Arpadi SM, Yin MT. Incident fractures in HIV-infected individuals: a systematic review and meta-analysis. AIDS. 2013; 27: 194957.

15. Dong HV, Cortés YI, Shiau S, Yin MT. Osteoporosis and fractures in HIV/hepatitis $\mathrm{C}$ virus coinfection: a systematic review and meta-analysis. AIDS 2014; 28: 2119-31.

16. Yeh MW, Ituarte $\mathrm{PH}, \mathrm{Zhou} \mathrm{HC}$, et al. Incidence and prevalence of primary hyperparathyroidism in a racially mixed population. J Clin Endocrinol Metab. 2013; 98: 1122-9.

17. Fraser WD. Hyperparathyroidism. Lancet. 2009; 374: 145-58.

18. Bilezikian JP, Brandi ML, Eastell R, et al. Guidelines for the management of asymptomatic primary hyperparathyroidism: summary statement from the Fourth International Workshop. J Clin Endocrinol Metab. 2014; 99: 3561-9.

19. Gunn IR, Gaffeny D. Clinical and laboratory features of calcium-sensing receptor disorders: a systematic review. Ann Clin Biochem. 2004; 41: 441-58.

20. Mollerup CL, Vestergaard P, Frøkjaer VG, et al. Risk of renal stone events in primary hyperparathyroidism before and after parathyroid surgery: controlled retrospective follow-up study. BMJ. 2002; 325: 807.

21. Vestergaard P, Mollerup CL, Frøkjaer VG, et al. Cohort risk of fracture before and after surgery for primary hyperparathyroidism. BMJ. 2000; 321: 598-602.

22. Canalis E, Mazziotti G, Giustina A, Bilezikian JP. Glucocorticoid-induced osteoporosis: pathophysiology and therapy. Osteoporos Int. 2007; 18: 1319-28.

23. Seibel MJ, Cooper MS, Zhou H. Glucocorticoidinduced osteoporosis: mechanisms, management, and future perspectives. Lancet Diabetes Endocrinol. 2013; 1: 59-70.

24. Yang IA, Clarke MS, Sim EH, Fong KM. Inhaled corticosteroids for stable chronic obstructive pulmonary disease. Cochrane Database Syst Rev. 2012; 7: CD002991.

25. Mattishent K, Thavarajah M, Blanco P, et al. Meta-review: adverse effects of inhaled corticosteroids relevant to older patients. Drugs. 2014; 74 : 539-47.

26. Loke YK, Cavallazzi R, Singh S. Risk of fractures with inhaled corticosteroids in COPD: systematic review and meta-analysis of randomised controlled trials and observational studies. Thorax. 2011; 66: 699-708.

27. Van Staa TP, Leufkens HG, Cooper C. The epidemiology of corticosteroid-induced osteoporosis: a meta-analysis. Osteoporos Int. 2002; 13: 777-87.

28. Kanis JA, Johansson H, Oden A, et al. A metaanalysis of prior corticosteroid use and fracture risk. J Bone Miner Res. 2004; 19: 893-9.

29. Heffernan MP, Saag KG, Robinson JK, Callen JP. Prevention of osteoporosis associated with chronic glucocorticoid therapy. JAMA. 2006; 295: 1300-3.

30. Homik J, Suarez-Almazor ME, Shea B, et al. Calcium and vitamin D for corticosteroid-induced osteoporosis. Cochrane Database Syst Rev. 2000; (2): CD000952.

31. Laatikainen AK, Kröger HP, Tukiainen HO, et al. Bone mineral density in perimenopausal women with asthma: a population-based cross-sectional study. Am J Respir Crit Care Med. 1999; 159: 1179-85.

32. Crawford BA, Liu PY, Kean MT, et al. Randomized placebo-controlled trial of androgen effects on muscle and bone in men requiring long-term systemic glucocorticoid treatment. J Clin Endocrinol Metab. 2003; 88: 3167-76.

33. Saag KG, Emkey R, Schnitzer TJ, et al. Alendronate for the prevention and treatment of glucocorticoid-induced osteoporosis. GlucocorticoidInduced Osteoporosis Intervention Study Group. N Engl J Med. 1998; 339: 292-9.

34. Reid DM, Hughes RA, Laan RF, et al. Efficacy and safety of daily risedronate in the treatment of corticosteroid-induced osteoporosis in men and women: a randomized trial. European corticosteroid-induced osteoporosis treatment study. J Bone Miner Res. 2000; 15: 1006-13. 
35. Reid DM, Devogelaer JP, Saag K, et al. Zoledronic acid and risedronate in the prevention and treatment of glucocorticoid-induced osteoporosis (HORIZON): a multicentre, double-blind, double-dummy, randomised controlled trial. Lancet. 2009; 373: 1253-63.

36. Homik J, Cranney A, Shea B, et al. Bisphosphonates for steroid induced osteoporosis. Cochrane Database Syst Rev. 2000; (2): CD001347.

37. Rossini M, Orsolini G, Viapiana O, et al. Bisphosphonates in the treatment of glucocorticoid-induced osteoporosis: pros. Endocrine. 2015; 49: 620-7.

38. Saag KG, Zanchetta JR, Devogelaer JP, et al. Effects of teriparatide versus alendronate for treating glucocorticoid-induced osteoporosis: thirty-sixmonth results of a randomized, double-blind, controlled trial. Arthritis Rheum. 2009; 60: 3346-55.

39. Dore RK, Cohen SB, Lane NE, et al. Effects of denosumab on bone mineral density and bone turnover in patients with rheumatoid arthritis receiving concurrent glucocorticoids or bisphosphonates. Ann Rheum Dis. 2010; 69: 872-5.

40. Nyandege AN, Slattum PW, Harpe SE. Risk of fracture and the concomitant use of bisphosphonates with osteoporosis-inducing medications. Ann Pharmacother. 2015; 49: 437-47.

41. Cosman F, de Beur SJ, LeBoff MS, et al. Clinician's guide to prevention and treatment of osteoporosis. Osteoporos Int. 2014; 25: 2359-81.

42. Mazziotti G, Canalis E, Giustina A. Drug-induced osteoporosis: mechanisms and clinical implications. Am J Med. 2010; 123: 877-84.

43. Kwok CS, Yeong JK, Loke YK. Meta-analysis: risk of fractures with acid-suppressing medication. Bone. 2011; 48: 768-76.

44. Abrahamsen B, Rubin KH, Eiken PA, et al. Characteristics of patients who suffer major osteoporotic fractures despite adhering to alendronate treatment: a National Prescription registry study. Osteoporos Int. 2013; 24: 321-8.

45. Turner MR, Camacho X, Fischer HD, et al. Levothyroxine dose and risk of fractures in older adults: nested case-control study. BMJ. 2011; 342: d2238.

46. Meier C, Kraenzlin ME, Bodmer M, et al. Use of thiazolidinediones and fracture risk. Arch Intern Med. 2008; 168: 820-5.

47. Miziak B, Blaszczyk B, Chroscinska-Krawczyk $\mathrm{M}$, et al. The problem of osteoporostic in epileptic patients taking antiepileptic drugs. Expert Opin Drug Saf. 2014; 13: 935-46.

48. Krol C, Dekkers M, Kroon M. Longitudinal changes in BMD and fracture risk in orthotopic liver transplant recipients not using bone-modifying treatment. J Bone Miner Res. 2014; 29: 1763-9.

49. Tufano A, Coppola A, Contaldi P, et al. Oral anticoagulant drugs and the risk of osteoporosis: new anticoagulants better than old? Semin Thromb Hemost. 2015; 41: 382-8.

50. Vanderschueren D, Laurent MR, Claessens F, et al. Sex steroid actions in male bone. Endocr Rev. 2014; 35: 906-60.

51. Shao YH, Moore DF, Shih W, et al. Fracture after androgen deprivation therapy among men with a high baseline risk of skeletal complications. BJU Int. 2013; 111: 745-52.

52. Hadji P, Gnant M, Body JJ, et al. Cancer treatment-induced bone loss in premenopausal women: a need for therapeutic intervention? Cancer Treat Rev. 2012; 38: 798-806.

53. Kwan ML, Lo JC, Tang L, et al. Bone health history in breast cancer patients on aromatase inhibitors. PLoS One. 2014; 9: e111477.

54. Zaman K, Thürlimann B, Huober J, et al. Bone mineral density in breast cancer patients treated with adjuvant letrozole, tamoxifen, or sequences of letrozole and tamoxifen in the BIG 1-98 study (SAKK 21/07). Ann Oncol. 2012; 23: 1474-81.

55. Diamond TH, Higano CS, Smith MR, et al. Osteoporosis in men with prostate carcinoma receiving androgen-deprivation therapy: recommendations for diagnosis and therapies. Cancer. 2004; 100: 892-9.

56. Hussain SA, Weston R, Stephenson RN, et al. Immediate dual energy Xray absorptiometry reveals a high incidence of osteoporosis in patients with advanced prostate cancer before hormonal manipulation. BJU. 2003; 92: 690-4.

57. Bouvard B, Hoppé E, Soulié P, et al. High prevalence of vertebral fractures in women with breast cancer starting aromatase inhibitor therapy. Ann Oncol. 2012; 23: 1151-6.

58. Coleman R, Body JJ, Aapro M, et al. Bone health in cancer patients: ESMO Clinical Practice Guidelines. Ann Oncol. 2014; 25: iii124-37.

59. Rizzoli R, Body JJ, Brandi ML, et al. Cancer-associated bone disease. Osteoporos Int. 2013; 24 : 2929-53.

60. Coleman R, de Boer R, Eidtmann H, et al. Zoledronic acid (zoledronate) for postmenopausal women with early breast cancer receiving adjuvant letrozole (ZO-FAST study): final 60-month results. Ann Oncol. 2013; 24: 398-405.

61. Gnant M, Pfeiler G, Dubsky PC, et al. Adjuvant denosumab in breast cancer (ABCSG-18): a multicentre, randomised, double-blind, placebo-controlled trial. Lancet. 2015; 386: 433-43.

62. Smith MR, Egerdie B, Hernández Toriz N, et al. Denosumab in men receiving androgen-deprivation therapy for prostate cancer. $\mathrm{N}$ Engl J Med. 2009; 361: 745-55.

63. Greenspan SL, Nelson JB, Trump DL, et al. Skeletal health after continuation, withdrawal, or delay of alendronate in men with prostate cancer undergoing androgen-deprivation therapy. J Clin Oncol. 2008; 26: 4426-34.

64. Forbes JF, Cuzick J, Buzdar A, et al. Effect of anastrozole and tamixifene as adjuvant treatament for early-stage breast cancer: 100-months analysis of the ATAC trial. Lancet Oncol. 2008; 9: 45-53.

65. Tentori F, McCullough K, Kilpatrick RD, et al. High rates of death and hospitalization follow 
bone fracture among hemodialysis patients. Kidney Int. 2014; 85: 166-73.

66. Kidney Disease: Improving Global Outcomes (KDIGO) CKD-MBD Work Group. KDIGO Clinical Practice Guideline for the Diagnosis, Evaluation, Prevention, and Treatment of Chronic Kidney Disease-Mineral and Bone Disorder (CKD-MBD). Kidney Int Suppl. 2009; 113: S1-130.

67. Chauhan V, Ranganna KM, Chauhan N, et al. Bone disease in organ transplant patients: pathogenesis and management. Postgrad Med. 2012; 124: 80-90.

68. Kulak CA, Borba VZ, Kulak J Jr, Custódio MR. Osteoporosis after transplantation. Curr Osteoporos Rep. 2012; 10: 48-55.

69. Dalle Carbonare L, Zanatta M, Braga V, et al. Densitometric threshold and vertebral fractures in heart transplant patients. Transplantation. 2011; 92: 106-11.

70. Giannini S, Sella S, Silva Netto F, et al. Persistent secondary hyperparathyroidism and vertebral fractures in kidney transplantation: role of calcium-sensing receptor polymorphisms and vitamin D deficiency. J Bone Miner Res. 2010; 25: 841-8.

71. Schousboe JT, Shepherd JA, Bilezikian JP, Baim S. Executive summary of the 2013 ISCD position development conference on bone densitometry. J Clin Densit. 2013; 16: 455-67.

72. Kanis JA, McCloskey EV, Johansson H, et al. Scientific advisory board of the european society for clinical and economic aspects of osteoporosis and osteoarthritis (ESCEO) and the committee of scientific advisors of the international osteoporosis foundation (IOF). European guidance for the diagnosis and management of osteoporosis in postmenopausal women. Osteoporos Int. 2013; 24: 23-57.

73. Cosman F, de Beur SJ, LeBoff MS, et al. Clinician's guide to prevention and treatment of osteoporosis. Osteoporos Int. 2014; 25: 2359-81.

74. Genant HK, Wu CY, van Kuijk C, Nevitt MC. Vertebral fracture assessment using a semiquantitative technique. J Bone Miner Res. 1993; 8: 1137-48.

75. Schousboe JT, Debold CR. Reliability and accuracy of vertebral fracture assessment with densitometry compared to radiography in clinical practice. Osteoporos Int. 2006; 17: 281-9.

76. Rossini M, Viapiana O, Idolazzi L, et al. Improvements in the management of rheumatic patients from vertebral image obtained through DXA. Reumatismo. 2006; 58: 253-60.

77. Ferrar L, Jiang G, Clowes JA, et al. Comparison of densitometric and radiographic vertebral fracture assessment using the algorithm-based qualitative (ABQ) method in postmenopausal women at low and high risk of fracture. J Bone Miner Res. 2008; 23: 103-11.

78. Bonnet N, Beneton M, Kanis JA, Charlesworth D. Vertebral fracture assessment (VFA) with a densitometer predicts future fractures in elderly women unselected for osteoporosis. J Bone Miner Res. 2008; 23: 1561-8.
79. Fuerst T, Wu C, Genant HK, et al. Evaluation of vertebral fracture assessment by dual X-ray absorptiometry in a multicenter setting. Osteoporos Int. 2009; 20: 1199-205.

80. Hospers IC, van der Laan JG, Zeebregts CJ, et al. Vertebral fracture assessment in supine position: comparison by using conventional semiquantitative radiography and visual radiography. Radiology. 2009; 251: 822-8.

81. Buehring B, Krueger D, Checovich M, et al. Vertebral fracture assessment: impact of instrument and reader. Osteoporos Int. 2010; 21: 487-94.

82. Jager PL, Jonkman S, Koolhaas W, et al. Combined vertebral fracture assessment and bone mineral density measurement: a new standard in the diagnosis of osteoporosis in academic populations. Osteoporos Int. 2011; 22: 1059-68.

83. Diacinti D, Del Fiacco R, Pisani D, et al. Diagnostic performance of vertebral fracture assessment by the lunar iDXA scanner compared to conventional radiography. Calcif Tissue Int. 2012; 91: 335-42.

84. El Maghraoui A, Rezqi A, Mounach A, et al. Systematic vertebral fracture assessment in asymptomatic postmenopausal women. Bone. 2013; 52: 176-80.

85. Diacinti D, Pisani D, D'Avanzo M, et al. Reliability of vertebral fractures assessment (VFA) in children with osteogenesis imperfecta. Calcif Tissue Int. 2015; 96: 307-12.

86. Rosen HN, Vokes TJ, Malabanan AO, et al. The official positions of the international society for clinical densitometry: vertebral fracture assessment. J Clin Densit. 2013; 16: 482-8.

87. Abrahamsen B, van Staa T, Ariely R, et al. Excess mortality following hip fracture: a systematic epidemiological review. Osteoporos Int. 2009; 20: 1633-50.

88. Watts NB, Adler RA, Bilezikian JP, et al. Osteoporosis in men: an Endocrine Society clinical practice guideline. J Clin Endocrinol Metab. 2012; 97: 1802-22.

89. Orwoll E, Ettinger M, Weiss S, et al. Alendronate for the treatment of osteoporosis in men. $\mathrm{N}$ Engl $\mathrm{J}$ Med. 2000; 343: 604-10.

90. Boonen S, Orwoll ES, Wenderoth D, et al. Onceweekly risedronate in men with osteoporosis: results of a 2-year, placebo-controlled, doubleblind, multicenter study. J Bone Miner Res. 2009; 24: 719-25.

91. Boonen S, Orwoll E, Magaziner J, et al. Onceyearly zoledronic acid in older men compared with women with recent hip fracture. J Am Geriatr Soc. 2011; 59: 2084-90.

92. Orwoll ES, Scheele WH, Paul S, et al. The effect of teriparatide [human parathyroid hormone (1$34)$ ] therapy on bone density in men with osteoporosis. J Bone Miner Res. 2003; 18: 9-17.

93. Langdahl BL, Teglbjærg CS, Ho PR, et al. A 24-month study evaluating the efficacy and safety of denosumab for the treatment of men with low bone mineral density: results from the ADAMO trial. J Clin Endocrinol Metab. 2015; 100: 1335-42. 
94. Kaufman JM, Audran M, Bianchi G, et al. Efficacy and safety of strontium ranelate in the treatment of osteoporosis in men. J Clin Endocrinol Metab. 2013; 98: 592-601.

95. Varenna M, Bertoldo F, Di Monaco M, et al. Safety profile of drugs used in the treatment of osteoporosis: a systematical review of the literature. Reumatismo. 2013; 65: 143-66.

96. Rossini M, Adami G, Adami S, et al. Safety issues and adverse reactions with osteoporosis management. Expert Opin Drug Saf. 2016; 15: 321-32.

97. Adami S, Romagnoli E, Carnevale V, et al. [Guidelines on prevention and treatment of vitamin D deficiency. Italian Society for Osteoporosis, Mineral Metabolism and Bone Diseases (SIOMMMS)]. Reumatismo. 2011; 63: 129-47. [Article in Italian]

98. Rossini M, Gatti D, Viapiana O, et al. Vitamin D and rheumatic diseases. Reumatismo. 2014; 66: 153-70.

99. Adami S, Giannini S, Bianchi G, et al. Vitamin D status and response to treatment in postmenopausal osteoporosis. Osteoporos Int. 2009; 20: 239-44.

100. Rossini M, Adami S, Viapiana O, et al. Dose-dependent short-term effects of single high doses of oral vitamin $\mathrm{D}(3)$ on bone turnover markers. Calcif Tissue Int. 2012; 91: 365-9.

101. Sanders KM, Stuart AL, Williamson EJ, et al. Annual high-dose oral vitamin D and falls and fractures in older women: a randomized controlled trial. JAMA. 2010; 303: 1815-22.

102. Institute of Medicine (US). Committee to review dietary reference intakes for vitamin D and calcium. In: Ross AC, Taylor CL, Yaktine AL, Del Valle HB, eds. Dietary reference intakes for calcium and vitamin D. Washington, DC: National Academies Press; 2011.

103. Holick MF1, Binkley NC, Bischoff-Ferrari HA, et al. Guidelines for preventing and treating vitamin D deficiency and insufficiency revisited. J Clin Endocrinol Metab. 2012; 97: 1153-8.

104. Rizzoli R, Boonen S, Brandi ML, et al. Vitamin D supplementation in elderly or postmenopausal women: a 2013 update of the 2008 recommendations from the European Society for Clinical and Economic Aspects of Osteoporosis and Osteoarthritis (ESCEO). Curr Med Res Opin. 2013; 29: 305-13.

105. Adami S, Bertoldo F, Brandi ML, et al. [Guidelines for the diagnosis, prevention and treatment of osteoporosis]. Reumatismo. 2009; 61: 26084. [Article in Italian]

106. Adami S, Bianchi G, Brandi ML, et al. Validation and further development of the WHO 10-year fracture risk assessment tool in Italian postmenopausal women: project rationale and description. Clin Exp Rheumatol. 2010; 28: 561-70.

107. Bonaccorsi G, Fila E, Cervellati C, et al. Assessment of fracture risk in a population of postmenopausal italian women: a comparison of two different tools. Calcif Tissue Int. 2015; 97: 50-7.

108. Adami S, Bertoldo F, Gatti D, et al. Treatment thresholds for osteoporosis and reimbursability criteria: perspectives associated with fracture risk-assessment tools. Calcif Tissue Int. 2013; 93: 195-200.

109. Yang SD, Chen Q, Wei HK, et al. Bone fracture and interaction between bisphosphonates and proton pump inhibitors: a meta-analysis. Int $\mathbf{J}$ Clin Exper Med. 2015; 8: 4899-910.

110. Black DM, Reid IR, Cauley JA, et al. The effect of 6 versus 9 years of zoledronic acid treatment in osteoporosis: a randomized second extension to the Horizon-Pivotal Fracture TRIAL (PFT). J Bone Miner Res. 2015; 30: 934-44.

111. Boonen S, Reginster J-Y, Kaufman J-M, et al. Fracture risk and zoledronic acid therapy in men with Osteoporosis. New Engl J Med. 2012; 367: 1714-23.

112. Varenna M, Adami S, Rossini M, et al. Treatment of complex regional pain syndrome type I with Neridronate: a randomized, double-blind, placebo controlled study. Rheumatology (Oxford). 2013; 52: 534-42.

113. Miller PD, Jamal SA, Evenepoel P, et al. Renal safety in patients treated with bisphopsphonates for osteoporosis: a review. J Bone Miner Res. 2013; 28: 2049-59.

114. Bedogni A, Campisi G, Fusco V, Agrillo A. Raccomandazioni clinico-terapeutiche su osteonecrosi dei mascellari associata a bisfosfonati e sua prevenzione. Padova: CLEUP; 2013.

115. Shane E, Burr D, Abrahamsen B, et al. Atypical subtrochanteric and diaphyseal femoral fractures: second report of a task force of the American society for bone and mineral research. J Bone Miner Res. 2014; 29: 1-23.

116. Rossini M, Lello S, Sblendorio I, et al. Profile of bazedoxifene/conjugated estrogens for the treatment of estrogen deficiency symptoms and osteoporosis in women at risk of fracture. Drug Des Devel Ther. 2013; 7: 601-10.

117. Sharifi M, Lewiecki EM. Conjugated estrogens combined with bazedoxifene: the first approved tissue selective estrogen complex therapy. Expert Rev Clin Pharmacol. 2014; 7 : 281-91.

118. Mirkin S, Ryan KA, Chandran AB, Komm BS. Bazedoxifene/conjugated estrogens for managing the burden of estrogen deficiency symptoms. Maturitas. 2014; 77: 24-31.

119. Valera MC, Gourdy P, Tremollieres F, et al. From the Women's Health Initiative to the combination of estrogen and selective ER modulators to avoid progestin addition. Maturitas. 2015; 82: 274-7.

120. Pinkerton JV, Harvey JA, Lindsay R, et al. Effects of bazedoxifene/conjugated estrogens on the endometrium and bone: a randomized trial. J Clin Endocrinol Metab. 2014; 99: E189-98. 